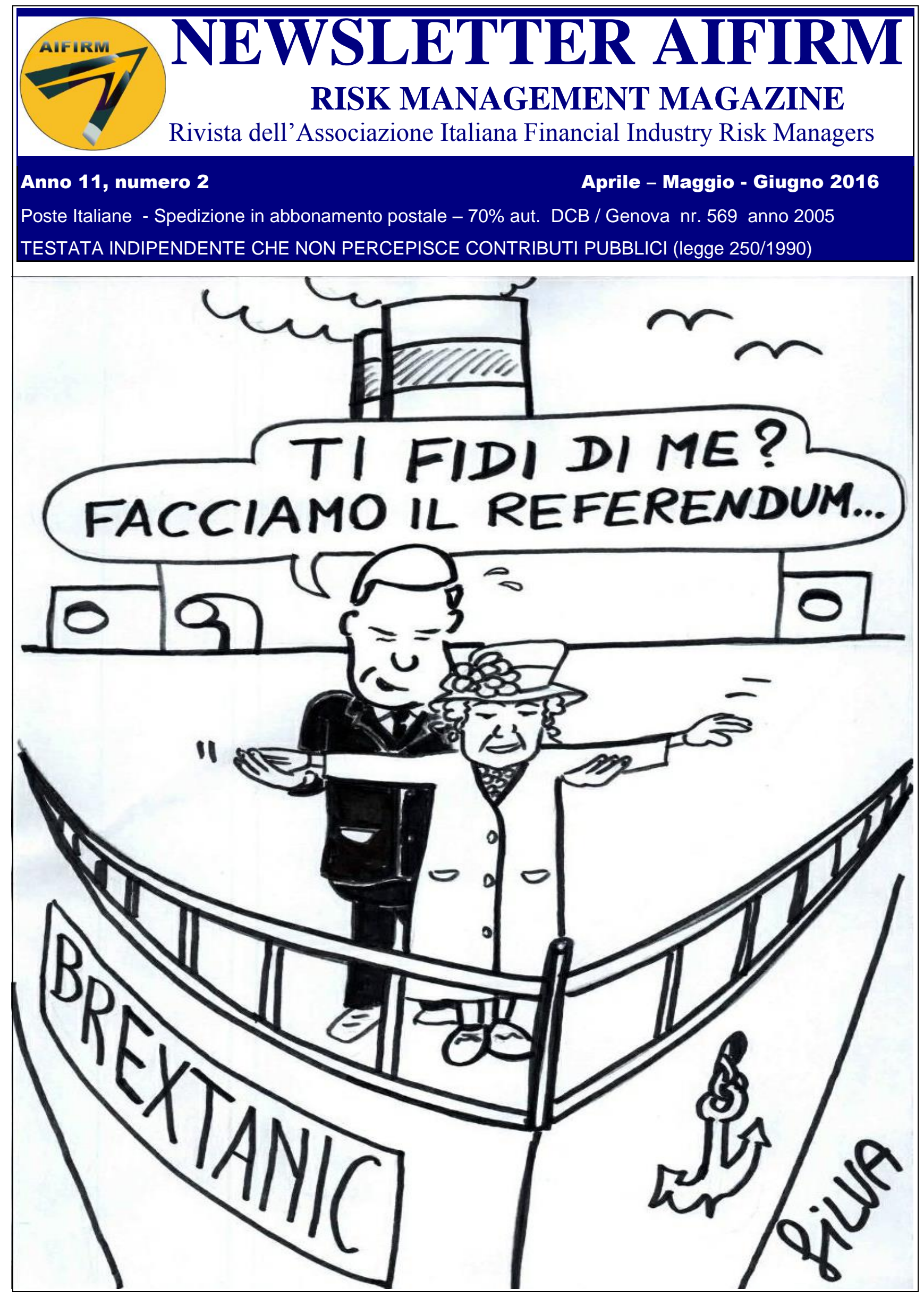

NEWSLETTER AIFIRM RISK MANAGEMENT MAGAZINE ANNO 11 № 2 - PAGINA - 1 - 


\section{IN QUESTO NUMERO}

\begin{tabular}{|c|c|}
\hline 3 & $\begin{array}{l}\text { Editoriale } \\
\text { di Maurizio Vallino }\end{array}$ \\
\hline 4 & $\begin{array}{l}\text { I nuovi coefficienti di ponderazione per il } \\
\text { calcolo del RWA nell'approccio standard } \\
\text { per i mutui residenziali - Proposta BIS } \\
\text { Camillo Giliberto e Fabio Salis }\end{array}$ \\
\hline 13 & $\begin{array}{l}\text { Il credito alle aziende non è ripartito, } \\
\text { contrariamente a quanto annunciato da un } \\
\text { anno } \\
\text { Antonio Pellegrini }\end{array}$ \\
\hline 18 & $\begin{array}{l}\text { Progettazione di una calibrazione robusta } \\
\text { per l'albero stocastico di Hull-White } \\
\text { mediante l'implementazione di euristiche } \\
\text { globali di ricerca } \\
\text { Pier Giuseppe Giribone, Simone Ligato, } \\
\text { Simone Fioribello }\end{array}$ \\
\hline
\end{tabular}

Processo di referaggio degli articoli proposti per la pubblicazione

Gli articoli che sono proposti alla rivista per la pubblicazione sono sottoposti in forma anonima a due sucessivi livelli di referaggio.

Il primo livello di referaggio (di ammissibilità) viene effettuato sull'articolo dai membri del Comitato di Direzione che ne valutano la conguità ai temi trattati dalla rivista.

Il secondo livello di referaggio (di pubblicabilità) viene effettuato sull'articolo da due referee scelti all'interno del Comitato di Direzione o all'esterno tra accademici, ricercatori, esperti della materia, che ne valutano il contenuto e forma.
Newsletter AIFIRM - Risk Management Magazine Anno 11, no 2 Aprile - Maggio - Giugno 2016

Direttore Responsabile:

Maurizio Vallino

\section{Condirettore}

Corrado Meglio

Comitato scientifico

Simona Cosma

Paola Ferretti

Giampaolo Gabbi

Andrea Giacomelli

Pier Giuseppe Giribone

Cristiana Schena

Giuseppe Torluccio

Enzo Scannella

Comitato di redazione: Emanuele Diquattro, Fausto Galmarini, Rossano Giuppa, Aldo Letizia, Paolo Palliola, Enzo Rocca, Fabio Salis

\section{Vignettista: Silvano Gaggero}

Proprietà, Redazione e Segreteria:

Associazione Italiana Financial Industry Risk

Managers (AIFIRM), Via Sile 18, 20139 Milano

Registrazione del Tribunale di Milano $n^{\circ} 629$ del $10 / 9 / 2004$

\section{ISSN 2283-7329}

\section{E-mail: segreteria@aifirm.it;}

Tel. 3896946315

Lunedì h.10-12; da Lunedì a Venerdì h.15-17

Stampa: Algraphy S.n.c. - Passo Ponte Carrega 62-62r 16141 Genova

Le opinioni espresse negli articoli impegnano unicamente la responsabilità dei rispettivi autori

SPEDIZIONE IN ABBONAMENTO POSTALE AI SOCI AIFIRM RESIDENTI IN ITALIA, IN REGOLA CON L'ISCRIZIONE 


\section{EDITORIALE}

Cari lettori,

la notizia della Brexit irrompe sulle agende dei politici, degli economisti, degli industriali e dei banchieri dell'Europa e del mondo: il cigno nero si è materializzato...

Se guardiamo ai modelli di risk management, l'esito del referendum inglese ci ha portato un livello di volatilità mai vista dei mercati finanziari e ha rimesso in discussione le tabelle di correlazione geo-settoriali del mondo del credito.

Il risk management ha sempre studiato i fenomeni di volatilità del passato per calibrare quell'iesimo percentile, coerente con il risk appetite della banca, che misurasse il livello di capitale da accantonare per ogni business. Le risultanze della Brexit, più di ogni altro fenomeno outlier antecedente, ci impongono una riflessione sulla tenuta dei modelli in un ambiente finanziario fortemente caratterizzato da scossoni che lo rendono sempre più volatile e imprevedibile.

Il fenomeno da studiare non è più la volatilità, bensì la volatilità della volatilità intesa come l'analisi dei repentini cambi di direzione dell'economia e della finanza.

Gli economisti prefigurano, a conseguenza della Brexit, un calo del PIL globale a partire da quello britannico, in gran parte dovuto all'apertura di una fase di incertezza che durerà anni, coerentemente ai tempi necessari per definire il divorzio tra il Regno Unito e l'Unione Europea.

Saprà l'Unione Europea recuperare parte del PIL perso sostituendosi a Londra nel ruolo di maggiore centro finanziario europeo? E' una partita aperta che vede Parigi e Francoforte in prima fila; ci auguriamo che Milano possa avere un ruolo non troppo defilato.

Maurizio Vallino 


\section{I nuovi coefficienti di ponderazione per il calcolo del RWA nell'approccio standard per i mutui residenziali - Proposta BIS}

di Camillo Giliberto (Banca MPS) e Fabio Salis (Banco Popolare) ${ }^{1}$

\section{Abstract}

The recent financial crisis has highlighted a series of problems in the operation, regulation and supervision of financial markets. The deteriorating credit quality of loans lately aroused considerable concern in several countries, especially in those most affected by the crisis, also encouraging the same regulators to improve sensitivity to the risks of banks and more evenly calibrate capital requirements. The aim of this work is to analyze and assess the impact that that would have both on the real estate market, and in terms of increased capital requirement with increasing risk (loan to value) with the Basel Committee's new proposal in December 2015 to introduce a new RWA for residential mortgages for banks that use a standard approach. The new rules proposed by the Basel Committee would permit to those banks that use a standard method for credit risk to have adequate capitalization and ensure greater stability of the financial system.

\section{Premessa}

Il Comitato di Basilea ha proposto a dicembre 2015, con il documento " Revisions to the standardised Approach for credit risk- second consultative document ${ }^{2}$ " di modificare le condizioni per la concessione dei prestiti per le banche che utilizzano una metodologia standard per il Credit Risk. All'esame, c'è un nuovo sistema di ponderazione del rischio dei mutui . L'obiettivo è quello di evitare una situazione analoga a quella verificatasi con i mutui subprime negli USA, concedendo prestiti a chi poi non è stato in grado di restituirli. Per evitare tutto ciò, il Comitato ha pensato di inasprire i criteri basati sulla proporzione fra prestito e valore dell'immobile (loan to value o ltv)

Alla luce della nuova proposta BIS, nei paragrafi successivi vengono illustrati ed analizzati gli aspetti più generali, di seguito sintetizzati:

- Crisi finanziaria e necessità di una nuova regolamentazione

- Norme vigenti e nuova proposta BIS (Revisions to the standardised Approach for credit risk- dicembre 2015)

- Principi di controllo del rischio immobiliare - Regolamento (UE) n 575/2013

- Ripresa del mercato immobiliare ed impatti con la nuova proposta BIS

- Maggiore aderenza al profilo di rischio per lo standardized approach ed allineamento ai rating interni

- Conclusioni

\section{Crisi finanziaria e necessità di una nuova regolamentazione}

La recente crisi finanziaria ha reso evidenti non solo rilevanti fattori di fragilità sistemica insiti nella struttura, nella composizione e nei modi di operare dei sistemi finanziari, ma anche alcune importanti criticità dell'impianto regolamentare di Basilea.

I criteri di calcolo dell'esposizione e dei coefficienti di ponderazione definiti da Basilea hanno sottovalutato i rischi degli impieghi di natura finanziaria. In particolare, le banche e i regolatori non avevano adeguatamente valutato il rischio e la crisi finanziaria che ha colpito l'economia globale. Le tensioni finanziarie hanno fatto emergere una serie di problemi nel funzionamento, nella regolamentazione e nella supervisione dei mercati finanziari. La stabilità finanziaria si è riproposta come obiettivo fondamentale della politica economica reale.

Il contesto finanziario ha evidenziato la necessità di integrare l'approccio della vigilanza microprudenziale, con una prospettiva macroprudenziale, che includa i rischi per la stabilità del sistema finanziario nel suo complesso. La crisi ha mostrato che le bolle immobiliari possono innescare elevati rischi per la stabilità del sistema finanziario, con significative ripercussioni negative sull'economia reale. Questo è soprattutto dovuto all'ampiezza del mercato immobiliare. In Italia, il comparto immobiliare, costituito da investimenti in costruzioni, spesa per affitti e servizi di intermediazione, raggiunge quasi un quinto del PIL ed il patrimonio globale delle famiglie italiane è costituito per oltre il 60 per cento dalle attività immobiliari. Il credito erogato al settore immobiliare sotto forma di prestiti alle imprese di costruzioni, servizi connessi con le attività immobiliari e l'erogazione di prestiti alle famiglie sotto forma di mutui ipotecari, hanno rappresentato, già prima dell’ultimo decennio, circa un terzo degli impieghi bancari totali.

Le considerazioni espresse sono frutto esclusivo del libero pensiero degli autori e non impegnano in alcun modo l'istituto di appartenenza

${ }^{2}$ Document consultative successivo al Revisions to the standardised Approach for credit risk (proposto a dicembre 2014) 
Nel nostro paese, dopo una forte espansione alla fine degli anni 90, il ciclo immobiliare ha invertito la tendenza ben prima della crisi globale del 2008-09 (Gobbi e Zollino, 2013). La durata e l'intensità della crisi hanno innescato condizioni di più elevata fragilità economico finanziaria e questo ha comportato per le banche un incremento dei prestiti deteriorati ed una minore capacità di concedere nuovi finanziamenti.

La consistenza dei crediti deteriorati in Italia, dopo aver raggiunto un picco a settembre 2015 (pari a 363 miliardi), alla fine del 2015 è risultata essere pari a $360^{3}$ miliardi (il 18,1\% del totale dei crediti verso la clientela) con un'incidenza sui prestiti al netto delle rettifiche di valore, pari al 10,8\% (4,8\% per le sole sofferenze).

Per agevolare lo smobilizzo dei crediti in sofferenza dai bilanci delle banche, sono stati effettuati degli accordi sulle sofferenze bancarie e nuovi incentivi potranno venire dallo schema di garanzia dello Stato sulle cartolarizzazioni di crediti in sofferenza (Gacs) e dalla nascita del fondo "Atlante".

\section{Norme vigenti e nuova proposta BIS (Revisions to the standaridsed Approach for credit risk- dicembre 2015)}

Negli ultimi anni, la maggiore volatilità del mercato immobiliare ha comportato maggiori rischi per le banche. Oggi, gli stessi istituti di credito non devono preoccuparsi solo di tenere sotto controllo il cliente, ma nell'eventualità che questi risulti insolvente, devono considerare anche l'andamento dei prezzi dell'immobile posto a garanzia. ll settore immobiliare italiano dopo un periodo di forte evoluzione ed espansione ha conosciuto in questi ultimi anni un pesante rallentamento di compravendite. Le banche italiane, anche se non pienamente colpite, hanno comunque risentito fortemente della crisi sistemica ed è quindi necessario anche per esse considerare il problema del rischio immobiliare e cercare di attenuarlo.

Le norme correnti, prevedono che gli immobili a garanzia delle esposizioni creditizie vengano valutati al valore di mercato. Al fine di poter verificare nel tempo il valore dell'immobile, è prevista un'adeguata sorveglianza sul bene immobile il cui valore deve essere verificato secondo le modalità seguenti:

- almeno ogni 3 anni per gli immobili residenziali o comunque per esposizioni superiori a 3 milioni di euro o al $5 \%$ del patrimonio di vigilanza della banca

- annualmente per le altre tipologie

- con maggiore frequenza in caso di variazioni significative delle condizioni di mercato.

Alla luce dei nuovi scenari economico-finanziari, il Comitato di Basilea ha proposto di introdurre tra le diverse modifiche, anche un nuovo sistema di ponderazione del rischio per le banche che utilizzano uno standard approach. In particolar modo, i regolatori attraverso il secondo documento di consultazione proposto a dicembre 2015 "Revisions to the standardised Approach for credit risk" prevedono di modificare il sistema di ponderazione dei mutui residenziali in quanto secondo gli stessi, la prassi attuale definita dal regolamento 575/2013 e dalla direttiva 2013/36/UE non sarebbe più adeguata per coprire gli intermediari finanziari dal rischio di default del debitore.

L'obiettivo principale della nuova proposta, è quello di migliorare la sensibilità ai rischi delle banche e di calibrare in modo più omogeneo i requisiti patrimoniali .

Attualmente, gli istituti di credito che adottano il metodo standard, utilizzano, ai fini del calcolo del RWA, per i mutui residenziali un unico fattore di ponderazione pari al 35\%. Per il futuro, viene proposta una ponderazione differenziata per LTV (Risk Drivers), secondo una griglia predefinita che prevede ponderazioni comprese tra il 25 e il $75 \%$ per le esposizioni immobiliari residenziali ( cfr. tab. 1a). Inoltre, viene proposta a causa di un rischio più elevato, una maggiore ponderazione per le stesse esposizioni immobiliari residenziali per le quali il rimborso dipende 'materialmente' dai flussi di cassa generati dai beni che garantiscono l'esposizione stessa (cfr. tab. 1c).

Di seguito vengono indicati, nelle tab. 1a ed 1b, i Risk Weight proposti e correnti:

Tab. 1a- Risk weight proposed for residential real estate exposures (Repayment is not materially dependent on cash flows generated by property)

\begin{tabular}{|c|c|c|c|c|c|c|}
\hline & LTV $<=40 \%$ & $40 \%<$ LTV $<=60 \%$ & $60 \%<$ LTV $<=80 \%$ & $80 \%<$ LTV $<=90 \%$ & $90 \%<$ LTV $<=100 \%$ & LTV $>100 \%^{4}$ \\
\hline Risk Weight & $25 \%$ & $30 \%$ & $35 \%$ & $45 \%$ & $55 \%$ & RW COUNTERPARTY $^{4}$ \\
\hline
\end{tabular}

\section{N. B: $\operatorname{LTV}>100 \%{ }^{4}$}

${ }^{3}$ Fonte Banca D'Italia

4 For residential real estate exposures to individuals with an LTV ratio higher than $100 \%$ the risk weight applied will be $75 \%$. For residential real estate exposures to SMEs with an LTV ratio higher than $100 \%$ the risk weight applied will be $\mathbf{8 5 \%}$. 
Tab. 1b- Risk weight current for residential real estate exposures (Repayment is not materially dependent on cash flows generated by prperty)

\begin{tabular}{|c|c|c|c|c|c|}
\hline & LTV $<=40 \%$ & $40 \%<$ LTV $<=60 \%$ & $60 \%<$ LTV $>=80 \%$ & $80 \%<$ LTV $<=90 \%$ & $90 \%<$ LTV $<=100 \%$ \\
\hline Risk Weight & $35 \%$ & $35 \%$ & $35 \%$ & $35 \%$ & $35 \%$ \\
\hline
\end{tabular}

Dal confronto delle tavole sopra riportate (Cfr. tab. 1a e 1b), si evince un risparmio di capitale solo per i mutui con rapporto tra finanziamento e valore della casa (loan to value) non superiore al $\mathbf{6 0 \%}$ (Cfr. tab.2), mentre si riscontraun aggravio di capitale solo per i mutui con LTV $>\mathbf{8 0 \%}$. Diversamente, rispetto alle norme attuali, non ci sarebbe nessun maggiore impatto di Capitale Assorbito per i Mutui con LTV compreso tra il 60\% e l' $80 \%$.

I minori requisiti patrimoniali richiesti per LTV non superiore al $60 \%$, potrebbero indurre gli stessi istituti di credito a restringere l'offerta del credito per LTV superiore al $60 \%$, incidendo in tal modo sia sull'offerta di credito che sulla domanda di equilibrio della clientela. L'effetto sul credito immobiliare potrebbe tuttavia essere inferiore alle attese: in un periodo di forte espansione del mercato immobiliare, le banche potrebbero trovare comunque profittevole continuare a erogare mutui, accrescendo gli aggregati creditizi, nonostante i maggiori requisiti patrimoniali ad esse richiesti.

Nella tab. 1c vengono indicati i nuovi coefficienti di ponderazione proposti per i mutui residenziali in cui il rimborso dipende dai flussi di cassa generati dall'immobile

Tab. 1c. - Risk weight table for residential real estate exposures (Repayment is materially dependent on cash flows generated by property)

\begin{tabular}{|c|c|c|c|}
\hline & LTV $<=60 \%$ & $60 \%<$ LTV $<=80 \%$ & LTV $>80 \%$ \\
\hline Risk Weight & $70 \%$ & $90 \%$ & $120 \%$ \\
\hline
\end{tabular}

Per quanto sopra riportato (cfr. tab. 1c), ABI non condivide, i nuovi coefficienti di ponderazione per LTV superiore al $60 \%$ e suggerisce di introdurre il Risk Weight della controparte come proposto e riportato nella tab. 1a (ovvero pari al $75 \%$ cfr. nota 4$)$

\section{Principi di controllo del rischio immobiliare - Regolamento (UE) $\mathbf{n}^{\circ}$ 575/2013}

Da Dicembre 2013, il Regolamento (UE) n575/2013 e la Direttiva 2013/36/UE conosciute rispettivamente come CRR e CRD IV i testi normativi sono stati recepiti a livello nazionale dalla Circolare 285 del 17 Dicembre 2013, promulgata dalla Banca d'Italia.

La presente Circolare raccoglie le disposizioni di vigilanza prudenziale applicabili alle banche e ai gruppi bancari italiani, riviste e aggiornate per adeguare la normativa interna alle novità intervenute nel quadro regolamentare internazionale con particolare riguardo al nuovo assetto normativo e istituzionale della vigilanza bancaria dell'Unione europea, nonché per tener conto delle esigenze emerse nell'esercizio della vigilanza sulle banche e su altri intermediari

Le circolari 263/2006 e $\mathbf{2 8 5 / 2 0 1 3}$ si poggiano su basi diverse. La prima deriva da una normativa nazionale, la seconda invece trae origine da una normativa sovranazionale.

La nuova regolamentazione lascia quindi poca discrezionalità nelle decisioni di vigilanza prudenziale alle singole Autorità di vigilanza nazionali, potere che, di fatto, passa principalmente nelle mani dell'ABE.

Il rischio immobiliare, alla luce della nuova regolamentazione, continua ad essere valutato all'interno del rischio di credito.

Si riportano di seguito, le nuove norme (cfr. regolamento 575/2013) che attualmente regolano le esposizioni creditizie garantite da garanzie immobiliari:

L'Art. 112 prevede che le varie esposizioni siano distinte in precise classi tra le quali c'è quella riguardante le esposizioni garantite da ipoteche su beni immobili.

L'Art. 124 procede poi affermando, anche a livello comunitario, la distinzione tra esposizioni garantite da immobili residenziali e da immobili non residenziali; per le prime la ponderazione è il $35 \%$ mentre per le seconde è il $50 \%$. Tuttavia la normativa comunitaria introduce una nuova condizione in cui le ponderazioni possono essere aumentate in entrambi i casi fino a un massimo di $\mathbf{1 5 0} \%$. Tale aumento è previsto in base a considerazioni relative alla stabilità finanziaria, ma soprattutto in base al livello delle perdite sulle esposizioni garantite da immobili e allo sviluppo del mercato dei beni immobili.

L'Art. 125 relativo agli immobili residenziali conferma le regole riportate nella circolare 263, ovvero, che la ponderazione al 35\% è attuabile se l'immobile è occupato o è destinato ad esserlo o se il proprietario lo ha locato. E' necessario che il valore dell'immobile posto in garanzia non dipenda dal merito di credito del debitore. 
Successivamente, si afferma che i flussi generati dal debitore non devono provenire in maggior misura dall'immobile e per questo gli enti creditizi sono liberi di utilizzare dei rapporti massimi di "mutuo concesso/ reddito percepito" per ovviare al problema. Si pone inoltre un limite quantitativo, in quanto, si afferma che la parte di esposizione a cui è assegnato il fattore di ponderazione del $35 \%$ non può essere superiore all'80\% del valore di mercato dell'immobile o al valore del credito ipotecario.

L'articolo infine si chiude descrivendo delle eccezioni alle precedenti condizioni. In particolare si dà la possibilità di derogare alla condizione secondo cui il reddito del debitore non è ricavato in misura prevalente dall'immobile. L'eccezione è consentita a patto che l'autorità competente dello stato dove è ubicato l'immobile abbia pubblicato delle prove a riguardo dello sviluppo del mercato immobiliare e di tassi di perdita contenuti. A differenza della normativa italiana, nel regolamento viene però anche chiaramente esposto cosa si intenda per tassi di perdita contenuti.

Il requisito è che in un qualsiasi anno le perdite derivanti dalle esposizioni garantite da immobili residenziali fino all' $80 \%$ del valore di mercato o di quello ipotecario non devono superare lo 0,3\% dei prestiti omologhi posti in essere.

Viceversa per le esposizioni garantite da immobili residenziali le perdite devono essere al massimo pari a $0,5 \%$ di tutte le esposizioni della stessa tipologia.

Tali vincoli devono valere per qualsiasi periodo, per cui se in anno uno dei due limiti non è rispettato cessa la possibilità di usufruire della deroga e torna a valere la norma generale.

L'Art. 126 esamina invece il caso degli immobili non residenziali. I requisiti quindi rimangono pressappoco gli stessi, per cui resta valido l'obbligo di uso o di futuro uso dell'immobile e la non correlazione tra capacità di rimborso del credito e valore dell'immobile. Ciò che cambia è invece la parte relativa alla ponderazione, che per gli immobili non residenziali sale a 50\%, e quella relativa ai limiti di esposizione garantita dall'immobile. Tali vincoli sono in questa seconda fattispecie più restrittivi, infatti, il fattore di ponderazione "agevolato" può essere applicato solo alla parte dell'esposizione che non supera il 50\% del valore di mercato dell'immobile o eventualmente il $60 \%$ del valore ipotecario sempre che l'autorità competente nazionale abbia stabilito rigorosi criteri per la definizione esatta del valore ipotecario.

Anche per gli immobili non residenziali vale poi la deroga alla parte della norma che vieta l'adozione del fattore di ponderazione ridotto se gli immobili in garanzia rappresentano la fonte principale di reddito del debitore. La deroga, ugualmente per come accade con gli immobili residenziali, è possibile solo se in nessun anno considerato le perdite derivanti da esposizioni garantite al massimo dal 50\% del valore dell'immobile - o il $60 \%$ del valore ipotecario - sono superiori a $0,3 \%$ e contemporaneamente se le perdite derivanti da esposizioni garantite da immobili non residenziali sono superiori a $0,5 \%$ del totale delle esposizioni omologhe. Con i tre articoli qui citati il regolamento definisce tutte le regole in materia di garanzie immobiliari se gli enti dovessero utilizzare il metodo standardizzato.

Il regolamento e quindi la circolare 285/2013 proseguono andando a definire tutte le regole per il metodo dei rating interni, ovvero il metodo IRB.

Per quanto riguarda le esposizioni garantite da immobili non si prevede la costituzione di un apposito portafoglio e non c'è nemmeno un sottogruppo della classe delle esposizioni al dettaglio come invece prevedeva la precedente circolare 263/2006. Tuttavia qualche cenno alle esposizioni garantite da immobili lo troviamo proprio all'interno delle norme relative alle esposizioni al dettaglio. In particolare, nel regolamento CRR, nell'Art. 154 che definisce il metodo di calcolo del fattore di ponderazione si afferma che l'indice di correlazione da usare nella formula è fissato a 0,15 .

Altro riferimento si ha all'Art. 164 che, invece, parla del metodo corretto di valutazione della LGD e dove si afferma che le esposizioni che non beneficiano di garanzie delle amministrazioni centrali non possono avere una LGD inferiore a $10 \%$ nel caso in cui siano garantite da immobili residenziali, o inferiore a $15 \%$ se garantite da immobili non residenziali.

Le autorità nazionali hanno poi la facoltà, qualora ce ne siano le ragioni, di poter aumentare tale percentuale per le esposizioni garantite da immobili ubicati nel loro stato, viceversa se sono ubicati in altro stato membro devono accettare i vincoli imposti dalle autorità locali.

\section{Ripresa del mercato immobiliare ed impatti con la nuova proposta BIS}

Nel 2015 ci sono stati segnali di ripresa per il mercato immobiliare italiano. Le compravendite sono aumentate del 7\%, grazie al calo del $2,5 \%$ dei prezzi delle case e alla crescita pari a c.a. il $97 \%$ (di cui le surroghe sul totale è pari, a c.a. il $31 \%$ - Fonte ABI) dei nuovi mutui erogati.

La maggiore proponsione degli istituti di credito alla concessione dei mutui è legata ai seguenti fattori:

- maggiore disponibilità, di liquidità da impiegare per la concessione di prestiti;

- decremento dei tassi di interesse, grazie all' intervento europeo (Quantitative Easing) che ha a ridotto i tassi di interesse sui prestiti. Una manovra studiata proprio per incentivare il credito alle famiglie e alle imprese e per contrastare lo spettro della deflazione (ossia della riduzione dei prezzi dovuta alla contrazione dei consumi); 
- minori rischi per le banche a seguito di una ripresa economica. Il PIL nl 2015 è aumentato dello 0,6\%, in crescita per la prima volta dal 2011.

La nuova ripresa economica nel corso del 2015, ha consentito alle banche di ridurre i rischi di credito nella concessione dei mutui (cfr. grafico 1 - Tassi di default Mutui a Privati).

Si riscontra infatti nell'ultimo anno, un decremento del tasso di default (cfr grafico 1), con un livello minimo pari a 1,7 registrato nel terzo trimestre del 2015, livello più basso degli ultimi 4 anni.

Grafico 1- Tassi di default Mutui a Privati

\section{Tasso di default Mutui a Privati}

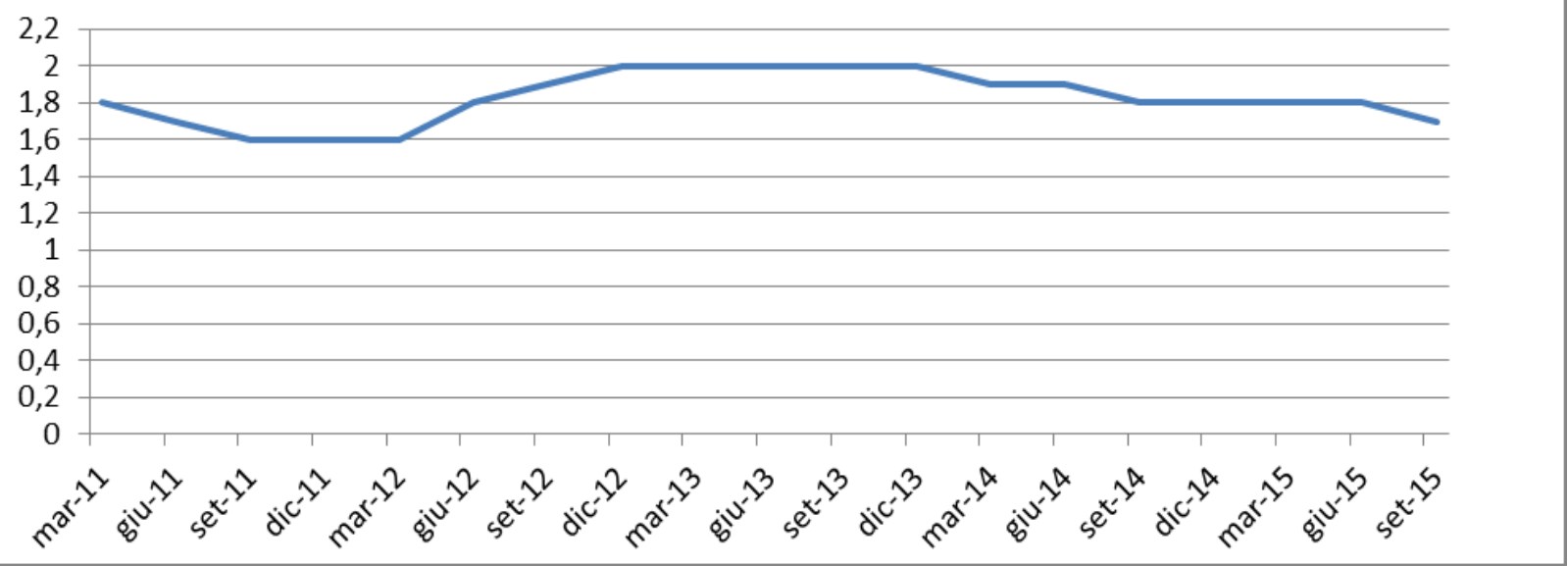

Si prevede inoltre una sensibile stima di crescita del PIL nei prossimi 10 anni con un recupero del PIL pre-crisi non prima del 2022 (cfr. Grafico 2-PIL pro-capite 2007-2026).

Grafico 2 - PIL pro-capite 2007-2026
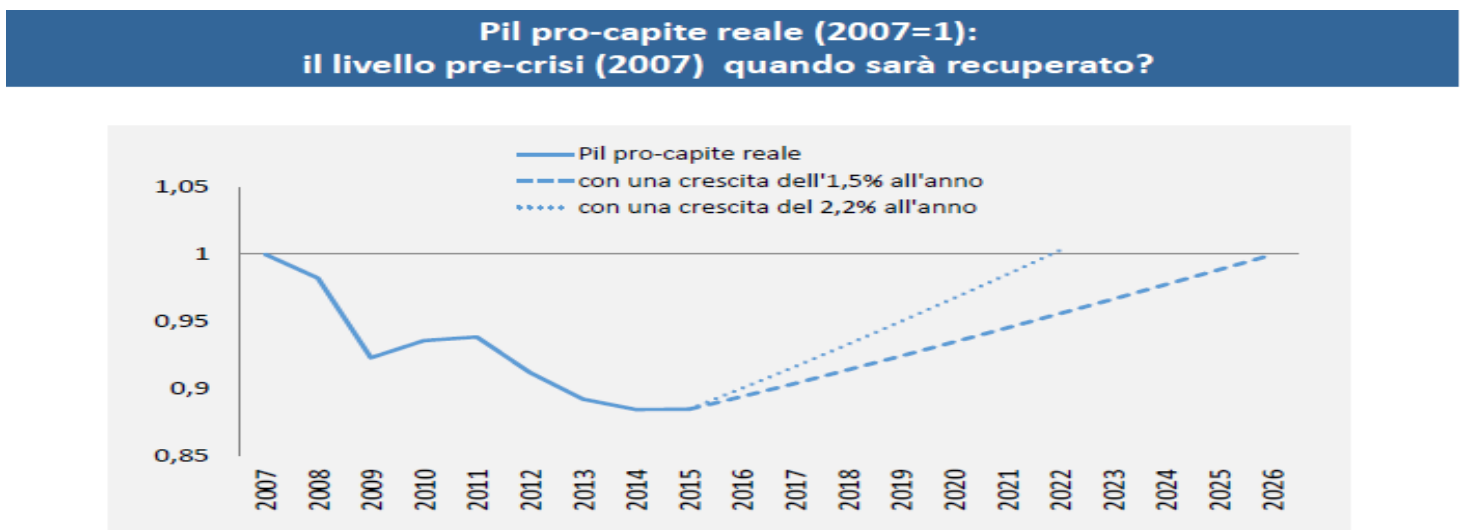

Fonte: elaborazioni Nomisma su dati Istat e stime Nomisma

La proposta del Comitato, secondo $\mathrm{ABI}^{5}$, favorirà prevalentemente l'erogazione dei mutui con il LTV inferiore al 60\%, che avranno accantonamenti più bassi. Questo pur comportando indubbi benefici per quanto concerne il sistema bancario (minori rischi), potrebbe compromettere la ripresa del mercato immobiliare (che nel corso del 2015 ha dato segnali di risveglio - cfr. grafico 4) in quanto comporterebbe:

(i) l'impossibilità da parte dei consumatori a "basso/medio reddito" di poter acquistare casa;

(ii) un aumento di tassi d'interesse per mutui con un LTV superiore al 60\%

${ }^{5}$ ABI Comments on Basel Committee on Banking Supervision Consultation Paper "Revision to the standardised approach for Credit Risk" 
La nuova normativa indurrebbe le banche ad avere una maggiore propensione commerciale a concedere mutui a basso LTV (non superiore al 60\%) e al contempo, a rinunciare ad un'opportunità di business , vista l'impossibilità da parte dei consumatori a "basso/medio reddito" di poter disporre di almeno il $40 \%$ di liquidità aggiuntiva per acquistare e/o ristrutturare casa.

L'Osservatorio sul credito al dettaglio realizzato da Assofin, Crif e Prometeia, ha evidenziato come nel 2015 il mercato del credito alle famiglie ha confermato i segnali di ripresa già registrati nella parte finale del 2014. L'aumento del reddito disponibile, la ripresa del mercato immobiliare e i bassi tassi di interesse applicati «hanno infatti contribuito alla ripresa della domanda di credito, mentre gli interventi di politica monetaria della Bce hanno sostenuto l'offerta».

Lo scorso anno, si è registrato un trend di decisa crescita del mercato dei mutui immobiliari sia nella componente dei mutui d'acquisto $(+21,3 \%)$ sia soprattutto in quella delle surroghe $(+780.6 \%)$.

Le previsioni indicano che per il biennio 2016-2017 il credito alle famiglie continuerà a crescere grazie al miglioramento delle condizioni economico-finanziarie delle famiglie, che potrebbe favorire l'acquisto di beni durevoli e gli investimenti immobiliari in parte rimandati durante gli ultimi anni»

I dati diffusi dall'Istat evidenziano nel 2015 una ripresa del mercato immobiliare dopo la serie negativa iniziata nel 2006, con un aumento delle compravendite del $\mathbf{+ 5 , 2 \%}$ sul $\mathbf{2 0 1 4}$. La ripresa risulta però limitata solo al comparto residenziale. Le convenzioni di compravendita di immobili ad uso abitazione ed accessori registrano infatti un aumento del $5,7 \%$ mentre quelle ad uso economico chiudono l'anno con un calo dell'1,3\%. Considerando tutti i comparti immobiliari, le grandi città vedono, infatti, un aumento tendenziale del 5,6\%, a fronte del $+4,8 \%$ dei piccoli centri.

Nel quarto trimestre 2015, il PIL dell'Italia (cfr. grafico 3) è cresciuto allo stesso ritmo del terzo trimestre $(0,3$ per cento sul trimestre precedente). Nello stesso periodo, le compravendite di abitazioni sono aumentate per il terzo trimestre consecutivo, determinando un rialzo del 6,5 per cento nella media del 2015 (2,9 nel 2014- cfr. grafico 4)

Grafico 3 - PIL Area Euro

Prodotto interno lordo dell'area dell'euro e dei principali paesi dell'area (1) (dati trimestrali; indici: $2005=100$ )

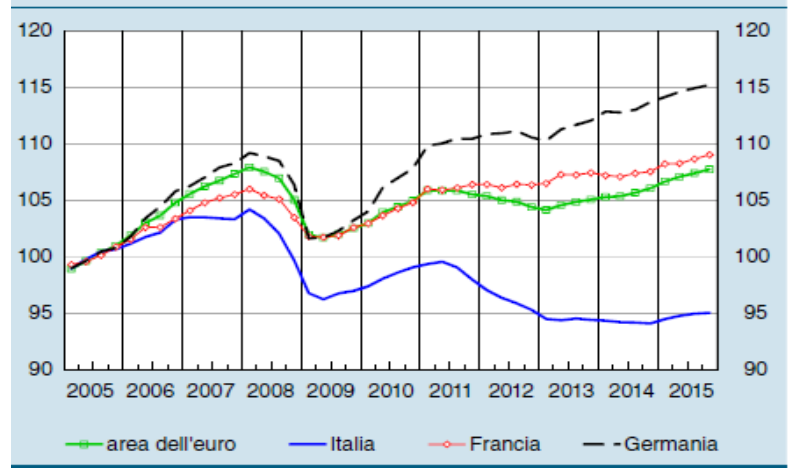

Grafico 4 - Compravendite e Prezzi delle abitazioni

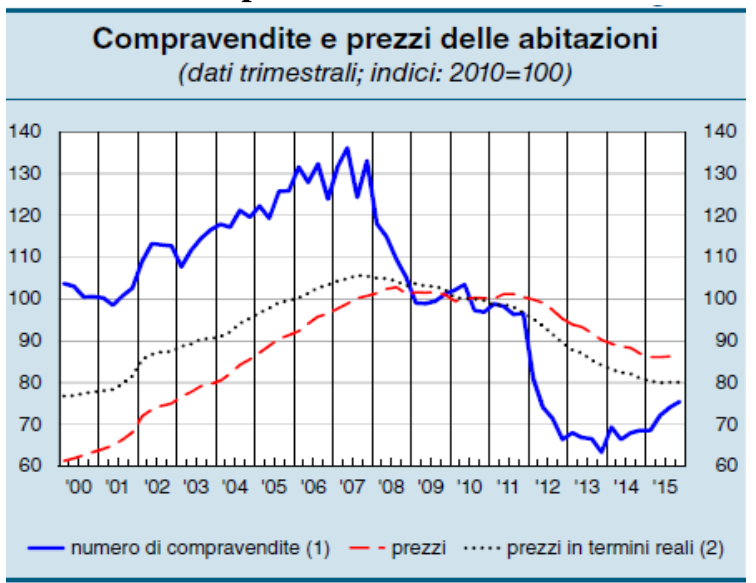

Fonte Banca D'Italia - Bollettino Economico 2-2016

\section{Maggiore aderenza al profilo di rischio per lo standardized approach ed allineamento ai rating interni}

La logica, della nuova proposta del Comitato di Basilea, è quella di attribuire un nuovo coefficiente di ponderazione crescente all'aumentare della stessa rischiosità, al fine di ottenere un requisito di capitale crescente all'aumentare del rischio delle attività.

La stima del nuovo RWA pertanto non sarà costante, ma sarà strettamente legata ai diversi coefficienti di ponderazione attribuiti a ciascun LTV (cfr. tab. 1a).

A titolo esemplificativo, si riportano, di seguito (cfr. tab. 2), i nuovi requisiti di capitale differenziati per LTV con i nuovi fattori di ponderazione proposti 
Tab. 2 - Requisiti di capitale- Mutui residenziali con fattore di ponderazione vigente e nuovo sistema di ponderazione proposto (cfr. tab. 1 punto a)

\begin{tabular}{|c|c|c|c|c|c|c|}
\hline \multicolumn{7}{|c|}{ Calcolo Requisito di Capitale - Approccio standard } \\
\hline A) Loan To Value & $39 \%$ & $60 \%$ & $70 \%$ & $80 \%$ & $90 \%$ & $100 \%$ \\
\hline B) Importo Erogato & $€ 100.000$ & $€ 100.000$ & $€ 100.000$ & $€ 100.000$ & $€ 100.000$ & $€ 100.000$ \\
\hline C) Nuovo fattore di ponderazione Proposto & $25 \%$ & $30 \%$ & $35 \%$ & $35 \%$ & $45 \%$ & $55 \%$ \\
\hline D) Fattore di ponderazione corrente & $35 \%$ & $35 \%$ & $35 \%$ & $35 \%$ & $35 \%$ & $35 \%$ \\
\hline E) Risk Weight Asset Proposto $=B^{*} \mathrm{C}$ & $€ 25.000$ & $€ 30.000$ & $€ 35.000$ & $€ 35.000$ & $€ 45.000$ & $€ 55.000$ \\
\hline F) RiskWeight Asset Corrente=B*D & $€ 35.000$ & $€ 35.000$ & $€ 35.000$ & $€ 35.000$ & $€ 35.000$ & $€ 35.000$ \\
\hline G) Requisito di capitale proposto $=8 \% * E$ & $€ 2.000$ & $€ 2.400$ & $€ 2.800$ & $€ 2.800$ & $€ 3.600$ & $€ 4.400$ \\
\hline H) Requisito di capitale corrente $=8 \% * F$ & $€ 2.800$ & $€ 2.800$ & $€ 2.800$ & $€ 2.800$ & $€ 2.800$ & $€ 2.800$ \\
\hline I) Delta Requisito di capitale proposto Vs. Requisito di capitale corrente & $-28,57 \%$ & $-14,29 \%$ & $0,00 \%$ & $0,00 \%$ & $28,57 \%$ & $57,14 \%$ \\
\hline
\end{tabular}

Dalla tab. 2 sopra riportata, si evince, un decremento di RC nel caso di LTV fino al $60 \%$, mentre si riscontra un aumento di RC nel caso in cui l'LTV sia superiore al 80\%. Il maggior rischio nel caso di Loan to Value superiore al 60\% (cfr. tab. 1a), comporterà uno spread più elevato da far pagare al richiedente ed una minore propensione a erogare i mutui .

Secondo i dati raccolti da CRIF, il livello di rischio di credito aumenta sensibilmente con un LTV superiore al' $80 \%$.

Per maggiori dettagli, si riportano nel Grafico 5 i tassi default rate Vs LTV:

Grafico 5 - Tassi di default Vs LTV

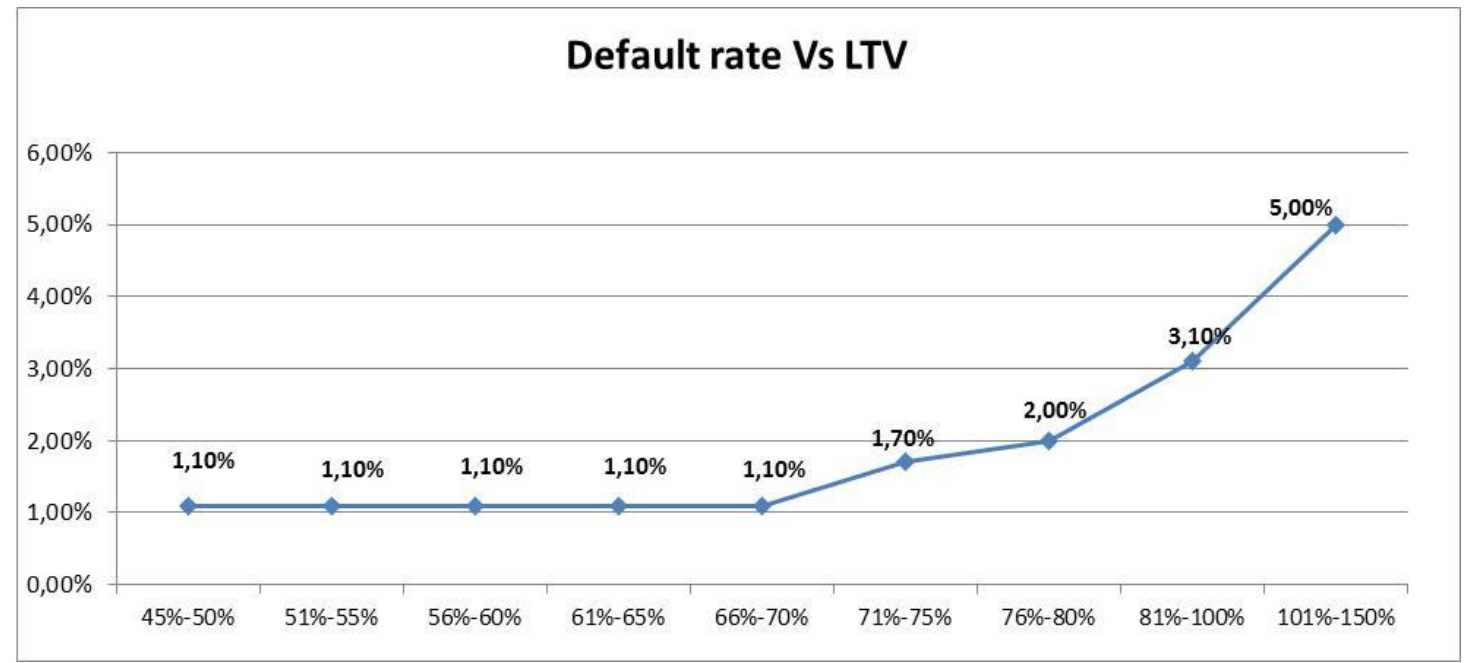

L'esperienza statistica sui default (cfr. grafico 5) sembra infatti confermare quanto indicato dallo stesso CRIF, che il rischio di credito aumenta in modo significativo quando il rapporto tra prestito e valore dell'immobile supera $1 ' 80 \%$.

Nel grafico 6 (sotto riportato), si propone un confronto fra le diverse metodologie di calcolo dei requisiti patrimoniali. Si nota da subito la maggiore flessibilità degli approcci avanzati rispetto a quello standardizzato. In particolare, è evidente che per le esposizioni dotate di un rating elevato, da AAA a BBB- l'utilizzo del metodo IRB avanzato porta ad un minore requisito rispetto al metodo standard mentre risulta essere più oneroso per esposizioni più rischiose con rating da $\mathrm{BB}+$ a $\mathrm{CCC}$ (con LGD pari al 35\%) e con rating da BB-a CCC (con LGD pari al 20\%). Il metodo IRB avanzato permette pertanto di ridurre ulteriormente la ponderazione se possono essere applicati valori di LGD inferiori, portando ad una migliore allocazione del capitale.

Il nuovo sistema di ponderazione proposto dai regulators tenderebbe ad allineare il metodo standard con il metodo interno dei rating avanzato. Il requisito di capitale nel metodo standard, non sarebbe più indifferenziato per ogni classe di rating (cfr. grafico 6), ma sarebbe stimato in funzione del LTV (cfr. tab. 1b) determinando pertanto un requisito di capitale crescente: maggiore sarà l'esposizione tra l'importo finanziato ed il valore dell'immobile (LTV), maggiore sarà il requisito di capitale richiesto. I maggiori requisiti di capitale richiesti , potrebbe indurre gli stessi istituti di credito ad un aumento dell'offerta di credito nei periodi di espansione ed una contrazione nei periodi di recessione acuendo la fase di credit crunch 


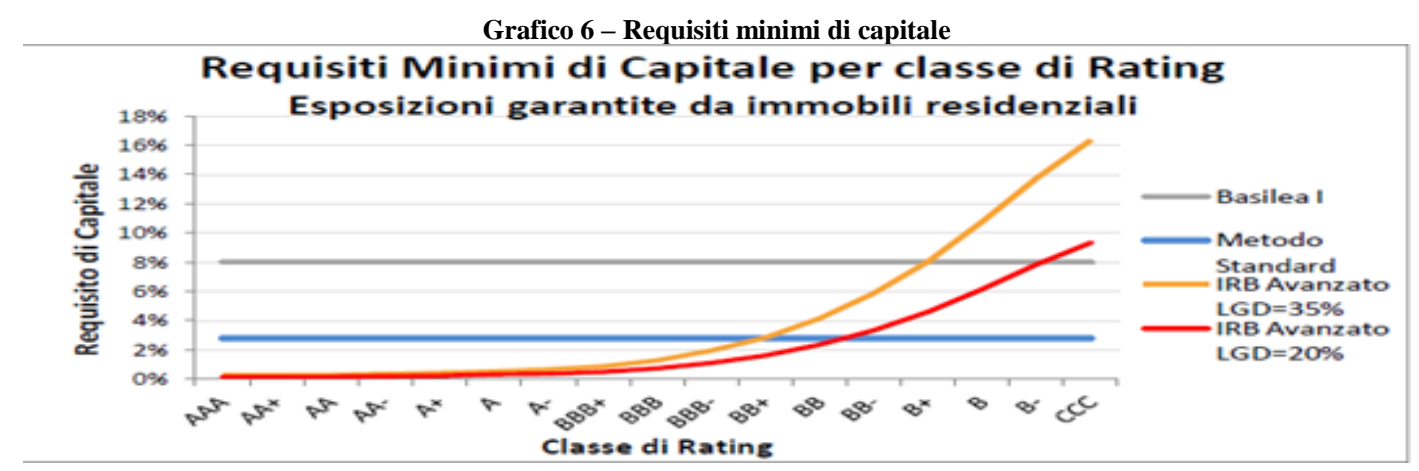

La nuova proposta del Comitato, consentirebbe anche alle banche che utilizzano un metodo standard, di applicare una forma di pricing "risk adjusted" ovvero un prezzo rettificato per il livello di rischio del cliente.

Il pricing differenziato per il rischio consentirebbe alle banche minori di avere i seguenti benefici:

- Politiche creditizie più mirate e premianti per i clienti più virtuosi, in grado cioè di migliorare il proprio merito creditizio, grazie anche ad una condivisione degli obiettivi comuni di stabilità e redditività;

- Attrazione dei clienti migliori proponendo prezzi più competitivi

- Accettazione di clienti meno virtuosi sostenendo la relativa rischiosità attraverso prezzi adeguati

Il pricing risk adjustment che ha l'obiettivo di preservare la redditività, si basa su un approccio EVA/Rik adjusted che remunera:

- $\quad$ il costo del capitale e gli accantonamenti

- $\quad$ il costo della liquidità

L'aumento degli spread per i debitori più rischiosi inciderebbe negativamente sui volumi erogati ai richiedenti con LTV $>60 \%$. Il più basso utilizzo di LTV in fase di erogazione del credito potrebbe rafforzare le banche in quanto la necessità di mantenere uno scarto tra il valore dei prestiti e quello delle garanzie consentirebbe di ridurre le perdite in caso di insolvenza dei prenditori. Nel contempo, riducendo la quota di debito finanziata da collaterale immobiliare, ne risulterebbe migliorata anche la solvibilità della clientela

\section{Conclusioni}

Negli ultimi anni il capitale ha assunto un'importanza centrale nelle politiche del sistema creditizio, internazionale. I rischi bancari, e le crisi che ne seguono, sono infatti sempre più diffusi e compito del capitale è proprio quello di "ammortizzare" future perdite che potrebbero minacciare l'esistenza stessa delle banche. Il peggioramento della qualità creditizia dei prestiti ultimamente ha destato preoccupazione in diversi paesi specie in quelli più colpiti dalla crisi. Dopo l'entrata in vigore della riforma di Basilea 3, che ha comportato un aumento della quantità e della qualità del capitale, l'intervento delle autorità si sta adesso concentrando sul denominatore dei coefficienti di adeguatezza patrimoniale, ovvero gli attivi bancari ponderati per il rischio. La nuova proposta presentata dei regulators, introduce un elemento" dinamico" rispetto alla situazione attuale: i requisiti patrimoniali a fronte dei mutui concessi alla clientela tenderanno a crescere all'aumentare del LTV ( il cosidetto Risk driver). La scelta del Loan to Value è centrale per classificare il debitore e lo scopo principale è quello di aumentare la risk sensitivity e garantire un'adeguata capitalizzazione e coerenza globale con le altre componenti del quadro di regolazione sul capitale. Al momento, la richiesta per modificare i coefficienti di ponderazione è ancora in fase di studio. Esistono delle perplessità in quanto il processo di regolamentazione proposto, potrebbe rallentare l'offerta del credito e rappresentare un elemento di freno della ripresa economia auspicata e sostenuta dalla Bce. 


\section{Bibliografia di riferimento}

- $\quad$ BIS - Revisions to the standaridsed Approach for credit risk

- $\quad$ ABI- Comments on Basel Committeeon Banking Supervsion Consultation Paper "Revision to the standardised approach for Credit Risk

- CRR- Regolamento 575/2013

- Banca D'Italia - Bollettino Ecoonomico 1/2016

- Banca D'Italia - Questioni di Economia e Finanza - Le misure Macroprudeniali introdotte in Europa per il settore Immobiliare di Daniele Ciani, Wanda Cornacchia e Paolo Garofalo

- Banca D'Italia- Circolare n. 285 del 17 dicembre 2013

- $\quad$ Banca D’Italia- Circolare n. 263 del 27 dicembre 2006 


\section{Il credito alle aziende non è ripartito, contrariamente a quanto annunciato da un anno.}

di Antonio Pellegrini

\section{Abstract}

Politicians, media, thematic experts, ordinary citizens should have greater respect and discernment in the use of official statistical data. If, in fact, it cannot prevent data from being interpreted differently by the various stakeholders, the bad practice to enter the media circuits misinterpretation of official data, passing it off as good, should be strongly opposed. Outside Italy, and particularly in the international arena, the Statistics receives more attention and consideration. In Italy, unfortunately, according to the author long experience, Statistic is often seen as subordinate and ancillary to the so-called queens' sciences. The article refuted a paradoxical argument put forward by the Italian Banking Association (ABI) during 2015, with the media support, that the credit crunch against enterprises is ended. Official data demonstrate that this thesis is definitely false.

\section{Articolo}

Un articolo del 2010, a firma del Prof. Enrico Giovannini - all'epoca Presidente dell' Istat $^{1}$, molto apprezzato per un'insolita onestà intellettuale nel commentare oggettivamente le statistiche ufficiali - esordiva con una domanda di sapore retorico: $<<$ Ma fra tanti problemi al mondo, l'ONU non aveva meglio da fare che celebrare la Giornata mondiale della statistica?>>.

Nell'articolo, la risposta seria veniva affidata al Presidente della Royal Statistical Society, secondo il quale la ragione per cui tutti pensano di poter trattare con superficialità i dati statistici risiede nel fatto che non si ha una chiara idea di quanto sia complesso e costoso produrre informazioni di buona qualità, attività per la quale è richiesta una elevata competenza professionale. Agli Statistici che si dedicano quotidianamente a tale attività, raccomandava di essere sempre più trasparenti e rigorosi, mentre a tutti gli altri utilizzatori - politici, media, esperti tematici, semplici cittadini - un maggior rispetto e discernimento nell'utilizzo dei dati statistici. Se, infatti, non si può evitare che i dati vengano interpretati differentemente dai diversi portatori d'interessi, va tuttavia combattuta la prassi perversa di immettere nei circuiti mediatici informazioni statistiche inaffidabili o costruite ad arte, spacciandole per buone. Erano e restano questi gli obiettivi della Giornata mondiale della statistica, che si celebra il 20 ottobre di ogni anno.

Fuori dall'Italia, ed in particolare in ambito internazionale, alla cui scuola mi sono formato professionalmente, la Statistica riceve maggiore attenzione e considerazione. In Italia, purtroppo, la Statistica è vissuta come subalterna ed ancillare rispetto alle c.d. scienze regine, spesso utilizzata in maniera strumentale da pseudo-statistici, $\mathrm{i}$ quali tendono a "torturare/massaggiare/nascondere" i dati elementari di base, fino a quando non ottengono "confessioni/risultati" conformi alle loro tesi precostituite. Un pessimo modo d'intendere la materia principe del metodo scientifico.

I progressi tecnologici e l'automazione dei processi di calcolo avvenuti nell'ultimo mezzo secolo, e che ho avuto l'opportunità di vivere intensamente, hanno consentito agli statistici di professione (Statistician) a dedicare molte più energie intellettuali alle metodologie ed all'analisi delle interconnessioni fra i fenomeni oggetto di studio, piuttosto che ai time-consuming calcoli manuali ${ }^{2}$. Un'evoluzione ampiamente prevista quasi tre secoli fa, come veniva riproposto mensilmente dalla copertina di "Cifre Essenziali" - una raccolta di statistiche ufficiali selezionate dall'Ufficio Studi della BNL degli anni ' 80 - attraverso la citazione di una frase "profetica" di Condorcet ${ }^{3}<<$ Ci andiamo ognor avvicinando all'epoca in cui sarà mestieri esporre per tavole lo stato delle nostre cognizioni $>$ >.

In generale, infatti, è possibile affermare che la profezia si è ampiamente realizzata, ma non laddove - come in Italia - prevale una cultura "classicheggiante", più incline ad apprezzare i grandi affreschi emotivi piuttosto che la cruda realtà desumibile dalla corretta interpretazione delle statistiche ufficiali. In tali situazioni accade spesso che a prevalere sia chi la "spara più grossa", o chi insiste per anni, anche attraverso accorgimenti pseudo-scientifici diversi, a sostenere false tesi.

Ho ritenuto opportuno iniziare con un lunga premessa, in quanto intendo confutare di seguito una di queste tesi, cercando per quanto possibile - di evitare l'impiego di formule matematiche, in modo che l'esposizione risulti comprensibile anche a coloro che hanno scarsa dimestichezza con i numeri. Mi riferisco alla tesi cangiante sul fenomeno del credit crunch nei confronti delle imprese minori in Italia, per anni testardamente negazionista, trasformata nel corso del 2015 in quella della "ripartenza a due cifre del credito alle imprese", una questione che sembra destinata a riproporsi anche nel 2016. Da qui il bisogno di un chiarimento, in quanto nell'ultima versione della tesi vengono citati dati ufficiali, il che impone chiarimenti preliminari di merito e di metodo.

Infatti, in una recente dichiarazione ufficiale dell'ABI, l'associazione delle banche italiane, apparsa su "la Repubblica" del 13 febbraio scorso, nel rispondere alla domanda se fosse terminata la fase di credit crunch anche per le imprese, così come già avvenuto per i mutui alle famiglie, la risposta "virgolettata" è stata la seguente: $<<$ Anche qui il dato di stock colloca a 793

\footnotetext{
${ }^{1}$ Oltre ad apprezzare lo spessore dell'insegnamento del Prof. Giovannini, del quale ho seguito un intero ciclo di lezioni tenute verso la metà degli anni ' 80 sul trattamento delle serie storiche economiche attraverso la tecnica della c.d. "analisi spettrale", ho anche avuto l'opportunità di avvalermi degli insegnamenti di altri Presidenti dell'Istat, fra cui Benedetto Barberi, Giuseppe de Meo, Vincenzo Siesto, e di altri cultori della scienza statistica, come Guglielmo Tagliacarne e Renato Guarini.

${ }^{2}$ Nessuna nostalgia per l'uso delle tavole logaritmiche o di altre "diavolerie" per velocizzare i calcoli manuali di prodotti o quozienti di numeri a più cifre. .

${ }^{3}$ Marie-Jean-Antoine-Nicolas Caritat, Marchese di Condorcet (1743-1794), filosofo, scienziato e uomo politico; studioso di matematica e scienze, nominato nel 1782 Segretario Perpetuo dell'Accademia di Francia. Autore della Relazione sull'Istruzione Pubblica, testo base per la pedagogia rivoluzionaria, che tuttavia non lo esentò dalla ghigliottina.
} 
miliardi di prestiti nel 2015, rispetto agli 808 del 2014. Ma quello di flusso registra circa un +11,5\%. Quindi anche il credito alle aziende è ripartito $>>$.

Chi ha buona memoria, o chi avesse la pazienza di recuperare le notizie apparse nel 2015 sui quotidiani economici, non avrebbe difficoltà nell'assimilare la situazione ai ripetuti - a cadenza mensile - falsi allarme "al lupo, al lupo" del pastore, con il rischio di imitarlo anche quando il lupo arriva davvero (che, nel nostro caso, è la vera ripresa, sempre più somigliante alla fantomatica "araba fenice").

In un precedente articolo, pubblicato su Dirigenza Bancaria, probabilmente ignorato dagli addetti ai lavori in quanto la Rivista ha una scarsa diffusione, ho tentato di chiarire i motivi della confusione prodotta da pseudo-statistici, i quali insistono nonostante l'ammonimento che "errare è umano, perseverare è diabolico" - nel sostenere che il credito alle imprese è ripartito, nonostante che le statistiche ufficiali dicano il contrario (da 808 miliardi euro si è scesi a 793 in un anno, ossia di ben 15 miliardi).

Siamo di fronte ad un classico paradosso, che tenterò - per l'ultima volta - di chiarire definitivamente.

Un chiarimento di merito: Le statistiche ufficiali sul credito bancario alle imprese riguardano sia le c.d. "Società non finanziarie" (alle quali si riferiscono i due dati di stock di prestiti bancari citati dall'ABI), sia le c.d. "Famiglie Produttrici", per le quali lo stock di prestiti è stato di 92 miliardi di euro nel 2015 e 93 nel 2014. Ne consegue che lo stock complessivo di prestiti alle imprese è stato pari a 901 miliardi di euro a fine 2014 e 886 miliardi a fine 2015, evidenziando una contrazione di 15 miliardi.

Due chiarimenti di metodo. Primo: In aggiunta alle statistiche ufficiali sugli stock di prestiti alle imprese, la Banca d'Italia pubblica anche i dati sui nuovi prestiti concessi nei vari mesi alle imprese (c.d. Erogazioni). A questo punto è sufficiente procedere con un semplice ragionamento, finalizzato a spiegare meglio la dinamica del fenomeno in esame, attraverso la costruzione del c.d. "balance sheet" relativo ai prestiti alle imprese. Infatti, analogamente a quanto avviene per la Popolazione in termini di stock e flussi, recentemente stimati dall'Istat e sintetizzati nel seguente prospetto

\begin{tabular}{|c|c|c|c|c|}
\hline STIME PER L'ANNO 2015 DELLA POPOLAZIONE RESIDENTE (in migliaia) \\
\hline Anno & $\begin{array}{c}\text { Popolazione } \\
\text { inizio anno }\end{array}$ & $\begin{array}{c}\text { Nati Vivi }+ \\
\text { Immigrati }\end{array}$ & $\begin{array}{c}\text { Morti }+ \\
\text { Emigrati }\end{array}$ & $\begin{array}{c}\text { Popolazione } \\
\text { fine anno }\end{array}$ \\
\hline 2015 & \multicolumn{6}{|c|}{60.795} & 733 & 872 & 60.656 \\
\hline FONTE: ISTAT - Indicatori demografici - 19 febbraio 2016 \\
\hline
\end{tabular}

anche per le serie storiche sui dati di stock e flussi lordi (in entrata ed in uscita) risulta vera l'identità

Stock Iniziale $_{t+1}=$ Stock Finale $_{t}$

Pertanto, anche se non viene pubblicato il dato sul flusso in uscita (c.d. Estinzioni), esso è ricavabile in maniera univoca, costruendo il balance sheet per ogni mese (tempo t):

Estinzioni $_{t}=$ Stock Iniziale $_{t}+$ Erogazioni $_{t}-$ Stock Finale $_{t}$

Secondo chiarimento di metodo: le variabili di stock e quelle di flusso sono - come noto - caratterizzate da dimensioni temporali diverse, in quanto le prime esprimono situazioni all'istante, mentre le seconde il movimento in un intervallo di tempo. Infatti, nel caso delle serie di stock il rapporto incrementale ha le dimensioni di una velocità (in crescita o decrescita), mentre l'analogo parametro per le serie di flusso, che esse stesse già hanno la natura di velocità, corrisponde meccanicamente al concetto di accelerazione o decelerazione. Nel primo caso si è nel campo della cinematica, mentre nel secondo in quello della dinamica, essendo associata all'esistenza di un'accelerazione/decelerazione. Pertanto dire che le erogazioni - che sono solo una delle due componenti di flusso - crescono ad un ritmo a due cifre, significa semplicemente che evidenziano un'accelerazione. Ma per un discorso compiuto sulle prospettive del fenomeno, occorre conoscere anche cosa succede alla componente negativa. Se questa accelera in misura superiore alle erogazioni, il risultato netto conduce ad una contrazione del credito, cosa che è avvenuta puntualmente negli ultimi quattro anni, come si evince dal grafico che segue. 


\section{BALANCE SHEET DEI PRESTITI BANCARI ALLE IMPRESE NON FINAZIARIE \\ FONTE: Banca d'Italia - Base Dati Statistica: TSC20300-VTI10300) \\ (Importi in milioni di euro)}

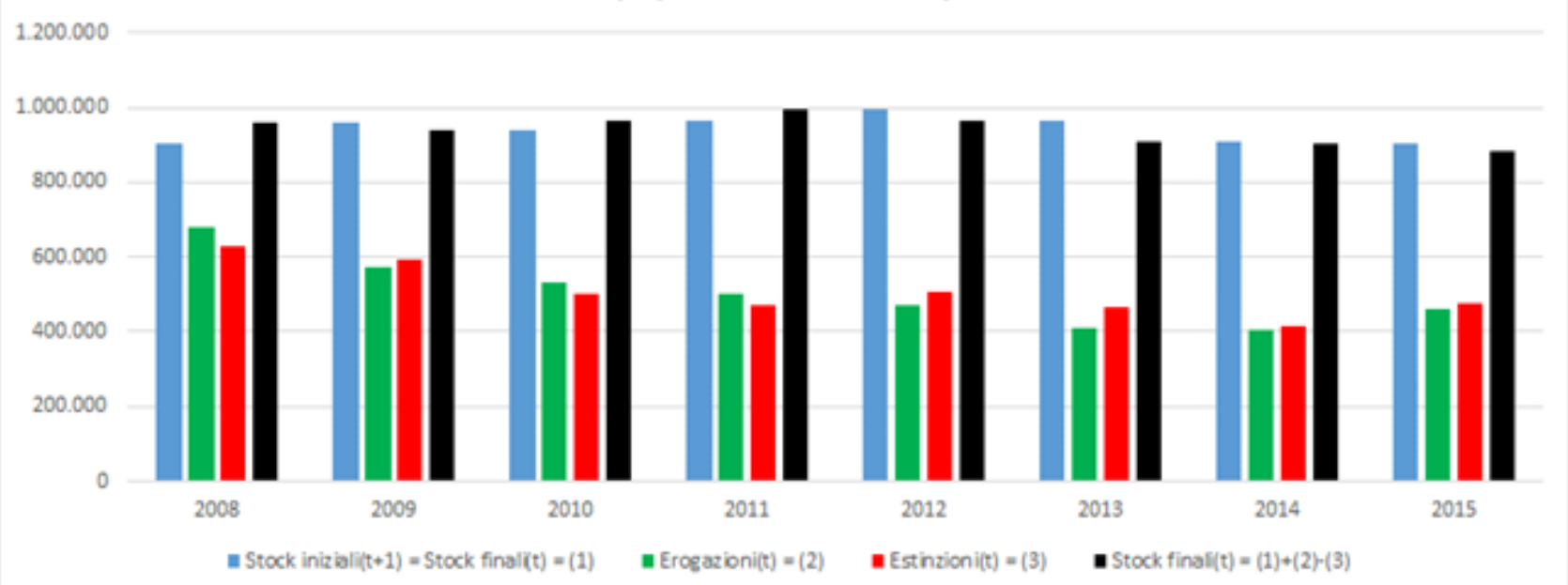

Se ne deduce che i prestiti bancari alle imprese nel 2015 sono diminuiti di oltre 15 miliardi di euro (-1,7\% su base annua), risultante da un'accelerazione dei nuovi prestiti ( $+13.6 \%$ su base annua) più contenuta rispetto a quella delle Estinzioni $(+15,1 \%)$, andandosi a cumulare per oltre 100 miliardi dal novembre 2011 .

Pertanto, l'informazione fornita dell'ABI, coerente con la tesi sostenuta nel corso del 2015, ossia che "il credito alle aziende è ripartito" è da rigettare, come dimostrano puntualmente le statistiche ufficiali riportate nella tavola che segue.

\begin{tabular}{|c|c|c|c|c|}
\hline \multicolumn{5}{|c|}{ BALANCE } \\
\hline & $\begin{array}{l}\text { Stock iniziali }(t+1)= \\
\text { Stock finali }(t)=(1)\end{array}$ & $\begin{array}{c}\text { Erogazioni }_{(t)}= \\
(2)\end{array}$ & $\begin{array}{c}\text { Estinzioni } i_{(t)}= \\
(3)\end{array}$ & $\begin{array}{c}\text { Stock finali }(t)= \\
(1)+(2)-(3)\end{array}$ \\
\hline 2008 & 903.856 & 681.434 & 626.741 & 958.549 \\
\hline 2009 & 958.549 & 573.915 & 592.529 & 939.935 \\
\hline 2010 & 939.935 & 529.875 & 503.905 & 966.533 \\
\hline 2011 & 966.533 & 501.445 & 473.225 & 994.753 \\
\hline 2012 & 994.753 & 473.177 & 505.423 & 962.507 \\
\hline 2013 & 962.507 & 410.677 & 463.906 & 909.277 \\
\hline 2014 & 909.277 & 407.237 & 415.302 & 901.212 \\
\hline \multirow[t]{2}{*}{2015} & 901.212 & 462.738 & 478.180 & 885.770 \\
\hline & $1 n_{1}$ & $\ldots \ldots \ldots \ldots$ CAGR & 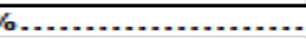 & (n) \\
\hline 2009 & 6,1 & $-15,8$ & $-5,5$ & $-1,9$ \\
\hline 2010 & $-1,9$ & $-7,7$ & $-15,0$ & 2,8 \\
\hline 2011 & 2,8 & $-5,4$ & $-6,1$ & 2,9 \\
\hline 2012 & 2,9 & $-5,6$ & 6,8 & $-3,2$ \\
\hline 2013 & $-3,2$ & $-13,2$ & $-8,2$ & $-5,5$ \\
\hline 2014 & $-5,5$ & $-0,8$ & $-10,5$ & $-0,9$ \\
\hline 2015 & $-0,9$ & 13,6 & 15,1 & $-1,7$ \\
\hline
\end{tabular}

A questo punto ritengo opportuno evidenziare, seguendo da anni un interessante grafico presente nei Bollettino Economico della Banca d'Italia, che dall'inizio della seconda ondata recessiva è stato modificato l'aggregato sottoposto a monitoraggio, da Prestiti Vivi, escludenti cioè le sofferenze ed i pronti contro termine, a Prestiti Totali. Una pessima ed incomprensibile notizia per gli statistici, che - come noto - preferiscono analizzare aggregati omogenei internamente, piuttosto che quelli più ampi, ma portatori di confusione interpretativa. Nella prima versione del grafico era più facile comprendere sia l'eventuale presenza del credit crunch nei confronti delle imprese, sia quella di una discriminazione dimensionale, come si intuisce osservando il grafico seguente, estratto dal BE di ottobre 2011. 


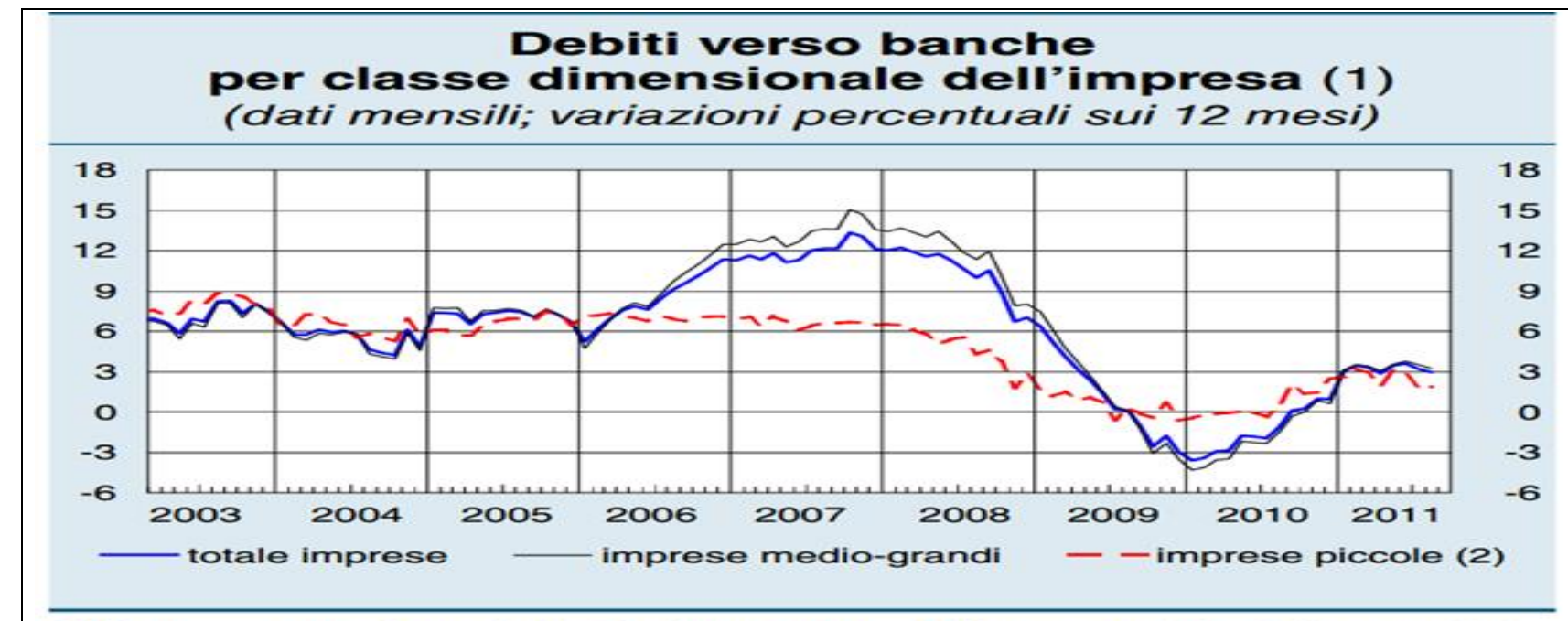

(1) Imprese non finanziarie. Dati corretti per l'effetto contabile delle cartolarizzazioni. I prestiti escludono i pronti contro termine, le sofferenze e alcune voci di minor rilievo che confluiscono nella definizione armonizzata dell'Eurosistema. I dati sono corretti per gli effetti di riclassificazioni, variazioni del cambio e altre variazioni non derivanti da transazioni. - (2) Società in accomandita semplice $e$ in nome collettivo, società semplici, società di fatto e imprese individuali con meno di 20 addetti.

Senza il cambiamento di definizione dell'aggregato, il grafico aggiornato sarebbe apparso come quello che segue, dalla cui lettura nessuno avrebbe potuto negare la persistenza del fenomeno del credit crunch nei confronti delle imprese nel corso del 2015, peraltro omogeneo per dimensione d'impresa, contrariamente a quanto propagandato dai media.

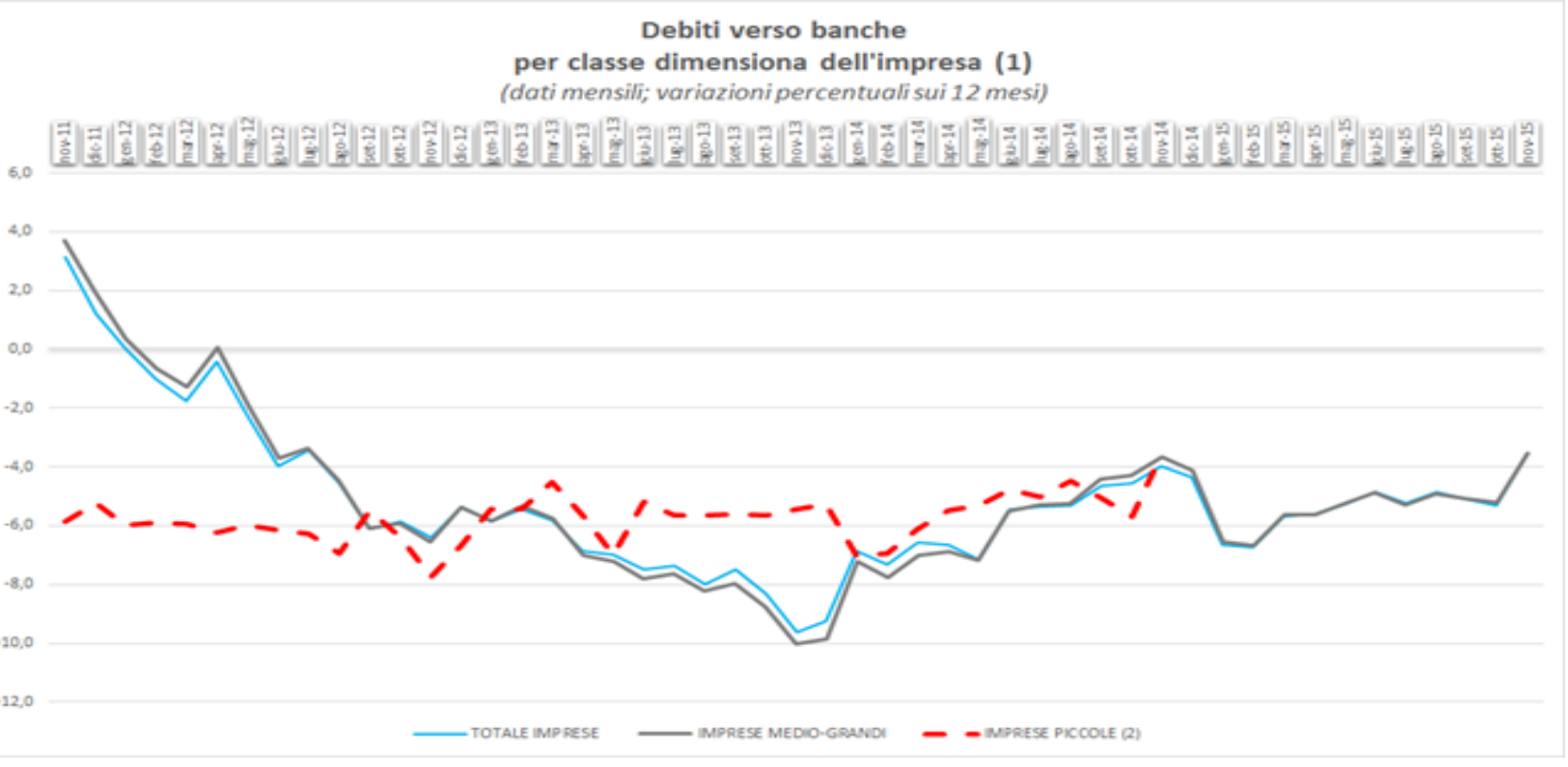

Ma cosa ancora più incomprensibile è la dichiarazione di "morte" anche per il grafico revisionato (ufficializzata dal Bollettino Economico di gennaio 2016), giustificata dalla possibile confusione con analoghi grafici sopravvissuti, e dalla presenza delle relative statistiche in una tavola nell'Appendice.

Dai due grafici esposti è possibile formulare una tesi alternativa a quella dell'ABI, che si fonda su tre pilastri:

1. Il credito alle imprese non è affatto ripartito, come analisi "filogovernative" vorrebbero far credere;

2. L'implementazione nel 2006 di Basilea2 ha introdotto elementi discriminatori, ai danni delle c.d. "imprese piccole", come paventato da alcuni rappresentanti del mondo dei Confidi e delle controgaranzie (nel convegno "Basilea 2 per le PMI: il futuro del credito alle PMI", organizzato il 2/3/2005 dalla Confesercenti di Brescia e dall'Associazione delle PMI a livello comunitario), nonostante la convergenza dei risultati di numerosi studi d'impatto, circa i vantaggi che Basilea 2 avrebbe apportato al mondo delle microimprese italiane (ben visibili nel primo grafico!);

3. Nel corso della seconda e prolungata crisi Il fenomeno del credit crunch ha interessato tutte le imprese, grandi e piccole. 
La tesi alternativa è la seguente: nelle fasi espansile del ciclo economico la propensione delle banche a erogare crediti alle imprese cresce più del dovuto e tende a discriminare le imprese di minore dimensione; nelle fasi recessive è il credit crunch a crescere più del dovuto, ma in maniera non discriminatoria. In tali circostante, al termine di un intero ciclo economico, il credito bancario alle imprese minori sarà inferiore a quello d'inizio ciclo: una morsa che tende a stringersi progressivamente nel tempo.

Un'ultima considerazione sulla tipologia e tempestività delle informazioni ufficiali per comparti in profonda trasformazione. Da tempo le BCC reclamano statistiche settoriali più specifiche per le loro esigenze di pianificazione strategica. Quanto alla tempestività, le statistiche ufficiali disponibili a marzo 2016 sul mercato dei Confidi sono ferme al 2014. E' come pretendere di guidate sull'autostrada avvalendosi soltanto di uno specchietto retrovisore "opaco".

Antonio Pellegrini 


\section{Progettazione di una calibrazione robusta per l'albero stocastico di Hull-White mediante l'implementazione di euristiche globali di ricerca}

di Pier Giuseppe Giribone (Banca Carige) - Simone Ligato (Banca Carige) - Simone Fioribello (Università di Genova)

\section{Abstract}

The calibration of Hull-White Tree is a crucial step for computing the fair value of complex financial instruments with interest rates as underlying. The tuning of characteristic parameters is performed using the Maximum Likelihood method, which is based on the minimization of the discrepancy between the model value and the market price of a vanilla derivative. This paper shows that the most popular commercial solvers are not always able to find the optimal values for the analyzed Markov Chain. The nature of this problem lies in the implementation of a proper optimization routine: the substitution of the traditional local search with a global heuristic makes the solver more robust.

This paper can be divided into four sections:

- The first part deals with the mathematical formulation of the minimum problem and it shows that a traditional approach is not always suitable.

- The second part shows the main differences between a traditional local search (Steepest Descent algorithm, Quasi-Newton method, Nelder-Mead simplex) and a global nature-inspired heuristic (Harmony Search, Cuckoo Search, Particle Swarm Optimization, Artificial Bee Colony, Firefly Algorithm, Simulated Annealing, Genetic Algorithm).

- The third section deals with the Matlab implementation of the meta-heuristics and their validation on the most popular benchmark surfaces.

- Application of the methodology

\section{Key Words:}

Hull-White Tree Calibration, Maximum Likelihood method, Global Optimization, Nature-Inspired Meta-heuristics, Harmony Search (HS), Cuckoo Search (CS), Particle Swarm Optimization (PSO), Artificial Bee Colony (ABC), Firefly Algorithm (FA), Simulated Annealing (SA), Genetic Algorithm (GA)

\section{Introduzione}

La calibrazione dei parametri caratteristici di un albero stocastico è un punto cruciale per la corretta determinazione del fairvalue di uno strumento finanziario complesso avente come sottostante i tassi di interesse. La taratura delle catene di Markov avviene comunemente applicando il principio di massima verosimiglianza, che si basa sulla minimizzazione della discrepanza tra il valore di modello ed il valore di mercato di uno strumento finanziario vanilla. Il presente studio dimostra che non sempre i più diffusi solver in commercio, basati su metodologie di ottimizzazione locali, sono in grado di affrontare tale problema in modo robusto e propone la sostituzione di questi metodi con l'implementazione di euristiche di ricerca globale. Tale sostituzione di algoritmo consente, pertanto, un incremento dell'affidabilità a fronte di un limitato aumento del tempo di calcolo.

Il paper può essere suddiviso in quattro sezioni:

- La prima parte presenta la formulazione matematica del problema di minimo da risolvere e dimostra come un approccio tradizionale può non essere sempre idoneo.

- La seconda parte mostra le principali differenze di funzionamento tra una metodologia di ricerca locale delle soluzioni (Steepest Descent algorithm, Quasi-Newton method, Nelder-Mead simplex) rispetto alle euristiche globali più popolari (Harmony Search, Cuckoo Search, Particle Swarm Optimization, Artificial Bee Colony, Firefly Algorithm, Simulated Annealing, Genetic Algorithm).

- La terza sezione si occupa dell'implementazione in Matlab delle meta-euristiche e della relativa validazione sui problemi di minimizzazione in applicazione alle più note superfici benchmark.

- Una volta testata l'affidabilità delle routine, si è proceduto alla calibrazione di un albero stocastico di Hull-White su un caso applicativo di mercato.

\section{Il problema algoritmico}

Si prenda in considerazione l'esempio inerente la calibrazione dell'albero di Hull-White riportato nella guida d'uso e manutenzione del software Matlab ${ }^{\circledR}$ - Financial Instruments [1], la cui implementazione è già stata trattata dagli autori nell'articolo relativo alla presentazione e all'applicazione dell'euristica Attraction Force Optimization (AFO) [2].

La formulazione matematica per il metodo di massima verosimiglianza, che consente di trovare i parametri caratteristici della catena markoviana (mean-reversion $\alpha$ e volatilità $\sigma$ ), è data da [3]:

$$
\min _{\alpha, \sigma} L(\alpha, \sigma)=\min _{\alpha, \sigma}[\text { ModelValue }(\alpha, \sigma)-\text { MarketValue }]^{2}
$$


L'obiettivo di tale calibrazione è quello di calcolare i valori di $\alpha$ e $\sigma$ in modo tale da minimizzare le differenze quadratiche ottenute confrontando il prezzo teorico del cap/floor (Model Value) con quello riscontrato dal mercato (Market Value).

La valutazione teorica dell'opzione scritta sul tasso è data dalle formule chiuse seguenti ${ }^{1}$ [4]:

$$
\begin{gathered}
\operatorname{Cap}(t, \tau, N, X)=N \sum_{i=1}^{n} P\left(t, t_{i-1}\right) \Phi\left(-h_{i}+\sigma_{p}^{i}\right)-\left(1+X \tau_{i}\right) P\left(t, t_{i}\right) \Phi\left(-h_{i}\right) \\
\operatorname{Floor}(t, \tau, N, X)=N \sum_{i=1}^{n}\left(1+X \tau_{i}\right) P\left(t, t_{i}\right) \Phi\left(h_{i}\right)-P\left(t, t_{i-1}\right) \Phi\left(h_{i}-\sigma_{p}^{i}\right) \\
\left\{\begin{array}{l}
\sigma_{P}^{i}=\sigma \sqrt{\frac{1-\exp \left[-2 \alpha\left(t_{i-1}-t\right)\right]}{2 \alpha}} B\left(t_{i-1}, t_{i}\right)=\sigma \sqrt{\frac{1-\exp \left[-2 \alpha\left(t_{i-1}-t\right)\right]}{2 \alpha}} \frac{1}{\alpha}\left\{1-\exp \left[-\alpha\left(t_{i}-t_{i-1}\right)\right]\right\} \\
h_{i}=\frac{1}{\sigma_{P}^{i}} \ln \frac{P\left(t, t_{i}\right)\left(1+X \tau_{i}\right)}{P\left(t, t_{i-1}\right)}+\frac{\sigma_{P}^{i}}{2}
\end{array}\right.
\end{gathered}
$$

$X$ rappresenta lo Strike dell'opzione;

$N$ corrisponde al valore nominale;

$n$ rappresenta il numero dei caplets/floorlets costituenti il cap/floor di riferimento;

$\tau$ è la frazione d'anno intercorrente tra un caplet (/floorlet) ed il successivo;

$P\left(t, t_{i}\right)$ è il fattore di sconto;

$\Phi(\cdot)$ è la funzione di distribuzione cumulata di una normale standardizzata;

$\alpha$ e $\sigma$ sono rispettivamente mean reversion e volatilità dell'albero di Hull-White, da determinare.

Si è pertanto proceduto alla taratura del HW Tree con i seguenti dati:

\begin{tabular}{|c|c|}
\hline \multicolumn{2}{|c|}{ Caratteristiche Finanziarie } \\
\hline Settle Date & $21 / 01 / 2008$ \\
\hline Maturity Date & $21 / 03 / 2011$ \\
\hline Strike Price & 0,069 \\
\hline Reset Frequency & Trimestrale \\
\hline Principal & 1000 \\
\hline Day Basis & ACT/ACT \\
\hline
\end{tabular}

\begin{tabular}{|c|c|c|}
\hline \multicolumn{3}{|c|}{ Strike/Volatilità di Black } \\
\hline Maturity & $5,9 \%$ & $7,9 \%$ \\
\hline $21 / 03 / 2008$ & 0,1533 & 0,1526 \\
\hline $21 / 06 / 2008$ & 0,1731 & 0,173 \\
\hline $21 / 09 / 2008$ & 0,1727 & 0,1726 \\
\hline $21 / 12 / 2008$ & 0,1752 & 0,1747 \\
\hline $21 / 03 / 2009$ & 0,1809 & 0,1808 \\
\hline $21 / 06 / 2009$ & 0,1809 & 0,1792 \\
\hline $21 / 09 / 2009$ & 0,1805 & 0,1797 \\
\hline $21 / 12 / 2009$ & 0,1802 & 0,1794 \\
\hline $21 / 03 / 2010$ & 0,1802 & 0,1733 \\
\hline $21 / 06 / 2010$ & 0,1757 & 0,1751 \\
\hline $21 / 09 / 2010$ & 0,1755 & 0,175 \\
\hline $21 / 12 / 2010$ & 0,1755 & 0,1745 \\
\hline $21 / 03 / 2011$ & 0,1726 & 0,1719 \\
\hline
\end{tabular}

\begin{tabular}{|c|c|c|}
\hline End Dates & Rates & Discount \\
\hline $21 / 03 / 2008$ & 0,0627 & 0,9898 \\
\hline $21 / 06 / 2008$ & 0,0657 & 0,9733 \\
\hline $21 / 09 / 2008$ & 0,0691 & 0,9555 \\
\hline $21 / 12 / 2008$ & 0,0717 & 0,9370 \\
\hline $21 / 03 / 2009$ & 0,0739 & 0,9182 \\
\hline $21 / 06 / 2009$ & 0,0755 & 0,8996 \\
\hline $21 / 09 / 2009$ & 0,0765 & 0,8815 \\
\hline $21 / 12 / 2009$ & 0,0772 & 0,8638 \\
\hline $21 / 03 / 2010$ & 0,0779 & 0,8462 \\
\hline $21 / 06 / 2010$ & 0,0783 & 0,8292 \\
\hline $21 / 09 / 2010$ & 0,0786 & 0,8127 \\
\hline $21 / 12 / 2010$ & 0,0789 & 0,7963 \\
\hline $21 / 03 / 2011$ & 0,0792 & 0,7802 \\
\hline $21 / 06 / 2011$ & 0,0793 & 0,7648 \\
\hline
\end{tabular}

Tabella 1. Caratteristiche finanziarie del Cap (sinistra)

Tabella 2. Struttura a termine della volatilità (centro)

Tabella 3. Struttura a termine dei tassi d'interesse (destra)

\footnotetext{
${ }^{1}$ Le formule (2) e (3) permettono di effettuare una calibrazione efficiente dei parametri che caratterizzano la catena di Markov analizzata. Queste equazioni sono rappresentative del contributo ModelValue $(\alpha, \sigma)$ presente in (1) e possono generalmente essere impiegate in contesti di tassi d'interesse negativi. Alternativamente all'utilizzo della (2) e (3) per la modellizzazione teorica del prezzo di cap/floor, può essere direttamente richiamata la costruzione dell'intero albero stocastico, scelta questa da considerarsi poco idonea per il maggiore tempo computazionale impiegato dal solver per la minimizzazione di $L(\alpha, \sigma)$.
} 
Applicando la routine standard di ottimizzazione gradientale locale (L-BFGS) per la minimizzazione della funzione $L(\alpha, \sigma)$, si ottengono i valori $\alpha=10^{-6}$ e $\sigma=0.0127$. Tale set di valori permettono di minimizzare la discrepanza tra $\mathrm{i}$ prezzi ottenuti con il modello teorico e quelli registrati dalle quotazioni di mercato ${ }^{2}$, come testimonia la figura 1.

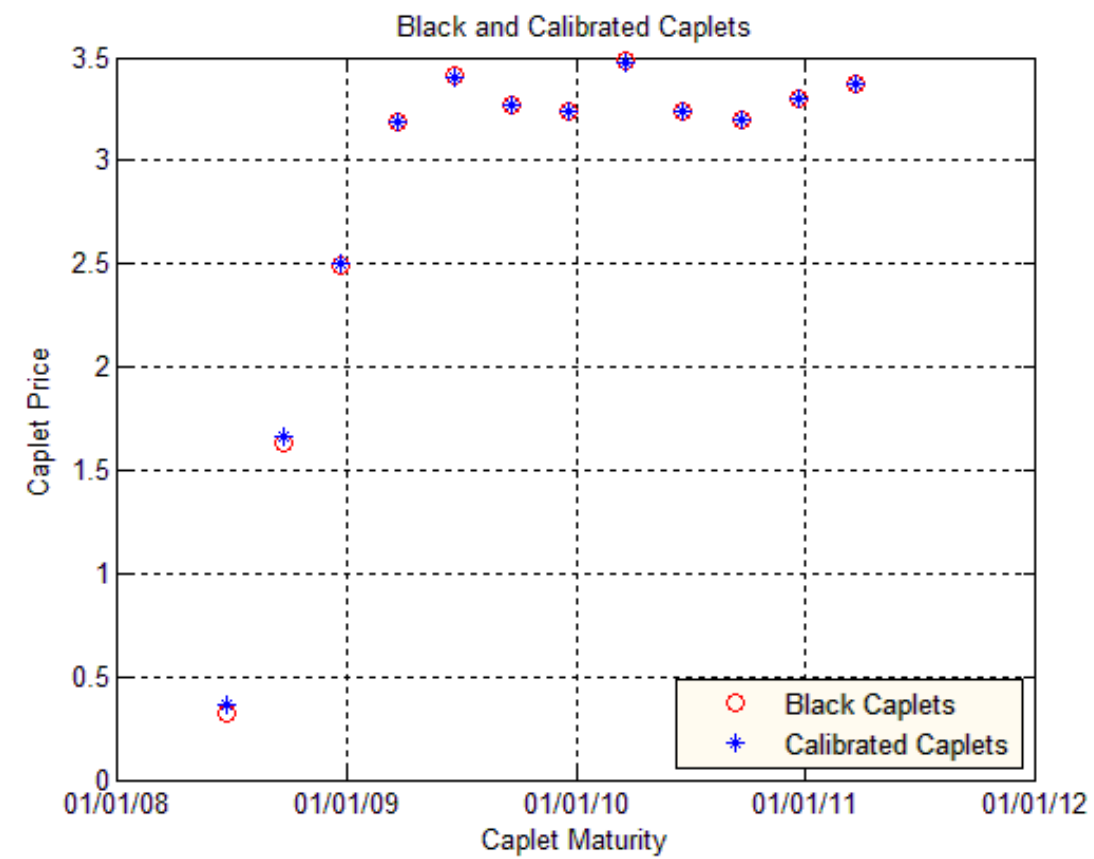

Figura 1. Black Caplets versus Calibrated Caplets (Strike 6.9\%) - routine di ottimizzazione locale gradientale

Con il medesimo codice, considerando un cap di riferimento con strike a $6.5 \%$, il metodo di ottimizzazione risulta non stabile ed i valori associati ai parametri liberi $(\alpha$ e $\sigma$ ) poco precisi, come testimoniato nella Figura 2.

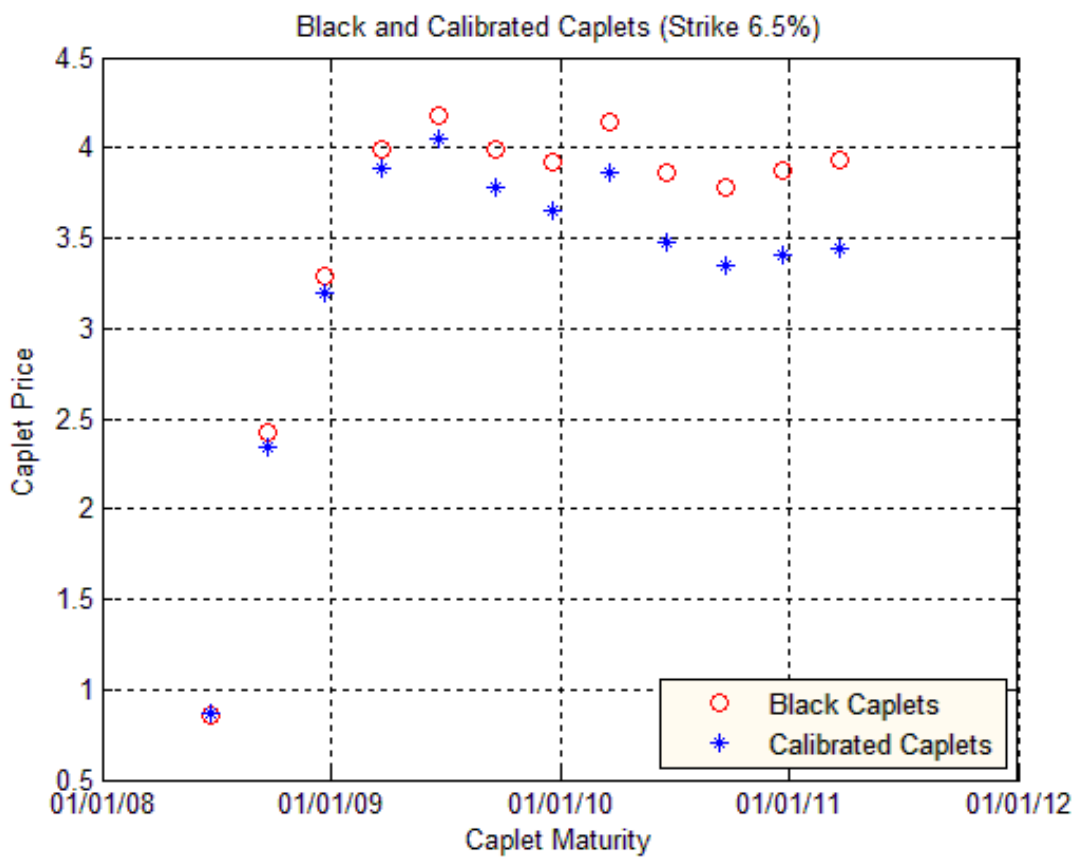

Figura 2. Black Caplets versus Calibrated Caplets (Strike 6.5\%) - routine di ottimizzazione locale gradientale

\footnotetext{
${ }^{2}$ Il contributo MarketValue della (1) rappresenta la quotazione di mercato dei cap/floor. Esistono principalmente due metodi per derivare questo dato: il primo modo (quello considerato dagli autori) si basa sul calcolo del valore delle opzioni sui tassi a partire dalle volatilità quotate sulla base della formula di Black '76; il secondo impiega direttamente le quotazioni dei premi messe a disposizione dai contributori. Generalmente, il primo fornisce MarketValue più regolari, ma non può funzionare in un contesto di tassi d'interesse negativi; il secondo invece, non usando formule di pricing proprie del BS-framework, funziona anche in contesti in cui il sottostante all'opzione non è positivo.
} 
Tale problema non è di natura finanziaria né inerente i dati d'ingresso del modello impiegato, ma è unicamente imputabile alla progettazione di una routine di ottimizzazione affidabile, robusta e di ricerca delle soluzioni di tipo "globale" e non "locale".

\section{Ottimizzazione locale versus globale}

Per ottimizzazione globale si intende il processo di ricerca del minimo (o analogamente del massimo) assoluto di una funzione di $n$ variabili, che possono essere eventualmente soggette a vincoli. In linea teorica, in assenza di vincoli, il problema può essere formulato come mostrato in [5]:

$$
\min _{\bar{x}} f(x)
$$

dove $f$ è la funzione obiettivo da minimizzare e il vettore $\vec{x}$ rappresenta le $n$ variabili indipendenti.

In particolare, se $f$ è una funzione $\mathfrak{R}^{n} \rightarrow \mathfrak{R}$, cioè tale che gli elementi $x_{i}$ del vettore $\vec{x}$ e il valore di output siano valori reali, il problema di ottimizzazione globale risulta essere definito nel continuo.

L'ottimizzazione globale di norma è un concetto diverso rispetto a quello di ottimizzazione locale. Un approccio locale consiste infatti in una ricerca di punti di ottimo locali, che rappresentano le soluzioni più "promettenti" all'interno di un sottoinsieme dell'intero dominio sperimentale di ricerca.

Un punto di ottimo locale $x^{*}$ può essere definito come un punto per il quale esiste almeno un $\delta>0$, per tutti i punti $x$ tali che $\left\|x-x^{*}\right\| \leq \delta$, risulti $f\left(x^{*}\right) \leq f(x)$. Sostanzialmente, in corrispondenza di un ottimo locale, il valore della funzione obiettivo $f\left(x^{*}\right)$ è minore o uguale a $f(x)$ per tutti gli altri punti che si trovano nella porzione di dominio analizzata.

Come esempio di problema di ottimizzazione globale, si può considerare la minimizzazione della funzione di Rastrigin in $x \in \mathfrak{R}^{n}:$

$$
f(x)=\sum_{j=1}^{n}\left(x_{j}^{2}-10 \cos \left(2 \pi x_{j}\right)+10\right)
$$

per $n=2$, rappresenta una delle funzioni benchmark tipicamente più utilizzate dai ricercatori per testare algoritmi di ottimizzazione (e sarà presentata anche nella sezione 4 di questo paper, come una delle 14 funzioni utilizzate per validare gli algoritmi di ottimizzazione globale implementati).

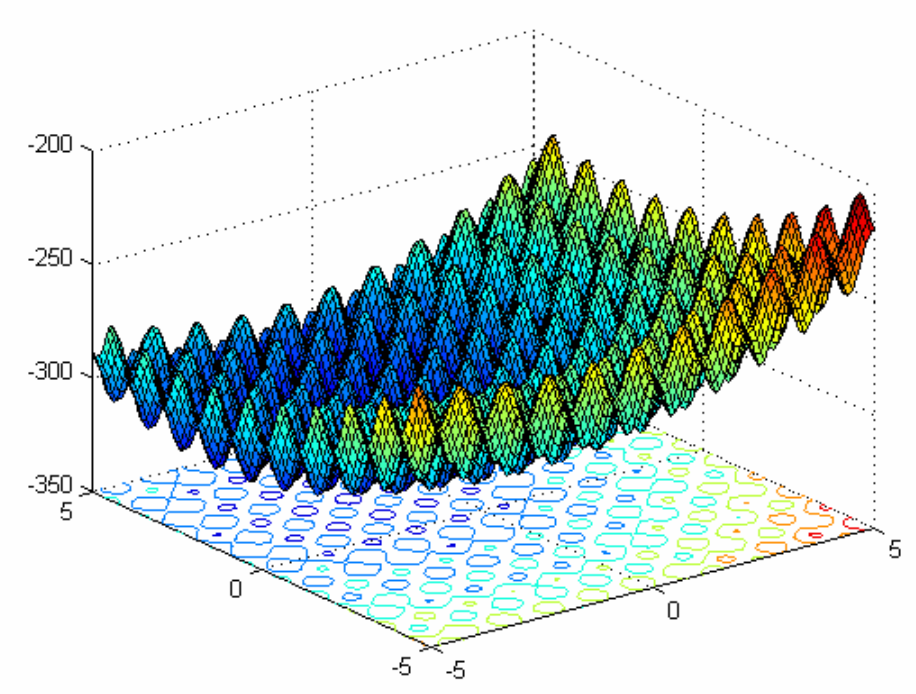

Figura 3. La superficie benchmark di Rastrigin

Come mostrato in Figura 3, la funzione di Rastrigin presenta un solo punto di minimo assoluto, ma è caratterizzata anche da numerosi minimi locali; le soluzioni ottenute applicando algoritmi di ottimizzazione locale generalmente non sono ottime in senso assoluto. E' preferibile ricorrere a tecniche globali, come mostrato nel prosieguo del lavoro. E' sconsigliabile affrontare tale difficile problema di ottimizzazione impiegando una ricerca di tipo locale poiché sarebbe impossibile raggiungere l'ottimo globale, ad esempio partendo dagli estremi del dominio di indagine: una maggiore probabilità di isolare il punto di minimo globale la si avrebbe implementando un'euristica globale. Tra le metodologie di ottimizzazione globale risultano di particolare interesse per la Comunità Scientifica le cosiddette tecniche "nature-inspired", in particolare, riferendosi metaforicamente al comportamento collettivo di cooperazione, lo scienziato Bonabeau ha definito la swarm intelligence come "il tentativo di progettare tecniche numeriche volte a simulare le intelligenze di gruppo osservabili in natura". In termini più generali si può 
impiegare il termine swarm per identificare un insieme ristretto di agenti "intelligenti ed indipendenti" che interagiscono, in vista di ottenere il raggiungimento di uno scopo comune (l'ottimo globale). Nonostante le tecniche di intelligenza artificiale seguano logiche di funzionamento diverse, vanno sottolineate le due proprietà fondamentali che caratterizzano qualsiasi struttura sociale organizzata, ovvero l'organizzazione individuale (self organization) e la suddivisione del lavoro (division of labour): tutti gli algoritmi di ricerca dell'ottimo devono tenere conto di questi aspetti, necessari per il corretto funzionamento della metaeuristica. Per self organization si intende un insieme di meccanismi dinamici, presenti nelle strutture di un sistema e usati come mezzo di comunicazione tra i componenti, e più in generale la proprietà di stabilire le regole base per lo scambio di informazione all'interno del gruppo. Con division of labour si fa riferimento alla specializzazione del lavoro all'interno di comunità strutturate.

Nella sezione 4, sono presentati i risultati ottenuti dall'applicazione delle più popolari tecniche di ottimizzazione globali ispirate ai processi naturali, che sono state testate su 14 funzioni benchmark. Le euristiche ${ }^{3}$ che sono state considerate sono: Harmony Search (HS, [6]), Cuckoo Search (CS, [7]), Particle Swarm Optimization (PSO, [8]), Artificial Bee Colony (ABC, [9]), Firefly Algorithm (FA, [10]), Simulated Annealing (SA, [11]) e i Genetic Algorithm (GA, [12]).

\section{Implementazione e validazione delle metodologie di ricerca globale}

Avendo sottolineato l'importanza di ricorrere ad euristiche di ottimizzazione globale, queste tecniche, sono state opportunamente implementate in ambiente Matlab ed il relativo codice è stato validato, al fine di valutarne la correttezza, mediante l'applicazione alle superfici benchmark $\mathrm{CEC} 05^{4}$, le cui espressioni analitiche sono presentate in [13].

In particolare, la validazione è stata effettuata testando le euristiche "nature-inspired" solo sulle funzioni non ibride, perché nel contesto applicativo di riferimento non si ricorre ad ibridazioni di funzioni obiettivo.

Le funzioni su cui sono state testate le euristiche sono:
1. Shifted Sphere Function
2. Shifted Schwefel's Function
3. Shifted Rotated High Conditioned Elliptic Function
4. Shifted Schwefel's Function with Noise in Fitness
5. Schwefel's Function with Global Optimum on Bounds
6. Shifted Rosenbrock's Function
7. Shifted Rotated Griewank's Function without Bounds
8. Shifted Rotated Ackley's Function with Global Optimum on Bounds
9. Shifted Rastrigin's Function
10. Shifted Rotated Rastrigin's Function
11. Shifted Rotated Weierstrass Function
12. Schwefel's Function
13. Expanded Extended Griewank's plus Rosenbrock's Function
14. Shifted Rotated Expanded Schaffer's

Le Figure 4-7 riportano quattro tra le superfici che sono state impiegate per la validazione delle routine di ricerca globale implementate (rispettivamente ID. 4 - 11 -12 - 14)

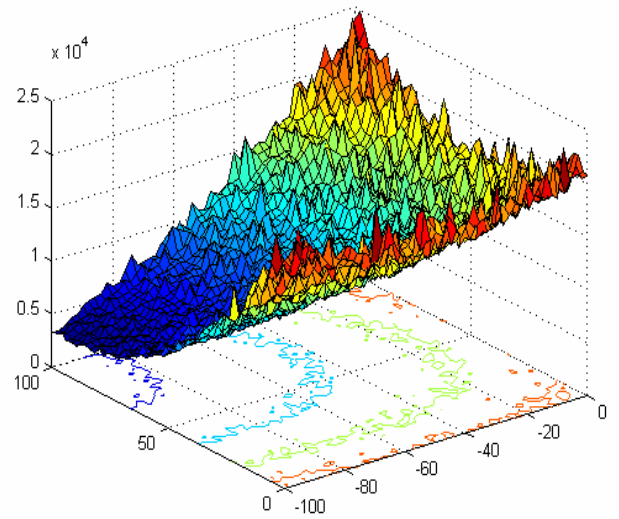

Figura 4. Shifted Schwefel's Function with Noise in Fitness

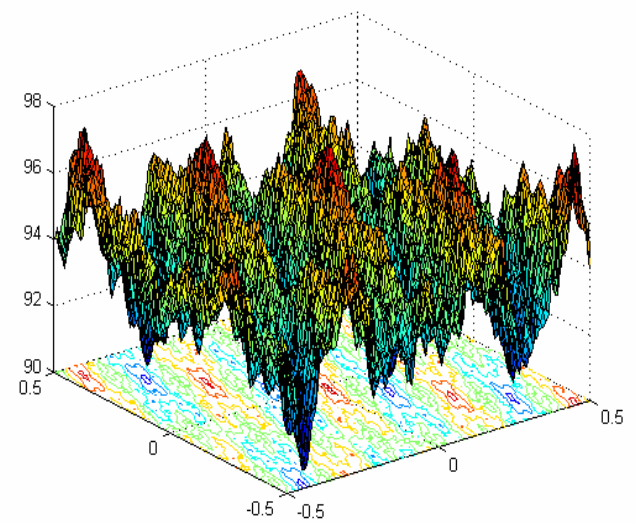

Figura 5. Shifted Rotated Weierstrass Function

${ }^{3}$ Il pseudo-codice di funzionamento delle meta-euristiche di ricerca globale non viene riportato per motivi di spazio. I lettori che fossero interessati ad approfondire l'argomento possono fare riferimento alla bibliografia specializzata [8], [9], [10], [11], [12].

${ }^{4}$ Le funzioni benchmark sono note agli Accademici del settore di ricerca operativa, poiché un test di ottimizzazione ad esito positivo condotte su di esse implica l'accettazione da parte della comunità scientifica di una nuova euristica di ottimizzazione, oppure la validazione di routine di minimizzazione, come nel caso del presente articolo. 


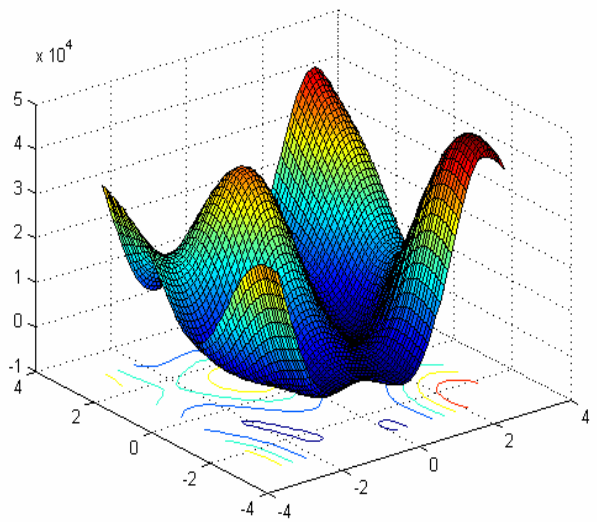

Figura 6. Schwefel's Function

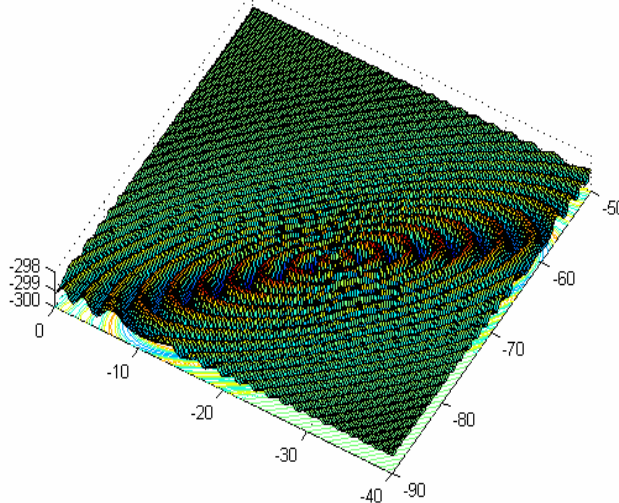

Figura 7. Shifted Rotated Expanded Schaffer's

Nella Tabella 4, per ciascuna delle 14 funzioni considerate, sono presentate le loro specifiche caratteristiche principali.

In generale, una funzione viene definita:

- Multimodal se presenta più punti di ottimo locale;

- $\quad$ Shifted se ha subito una traslazione verticale, del tipo $f(x) \pm c$;

- Separable se, considerando una funzione di due variabili indipendenti, questa può essere espressa come prodotto di due funzioni, ciascuna delle quali dipendente solo da una delle due variabili;

- Scalable se può essere oggetto di una trasformazione lineare che ne aumenta o ne diminuisce le dimensioni secondo un determinato fattore di proporzionalità, che è il medesimo in tutte le direzioni;

- $\quad$ Rotated se ha subito una trasformazione tale che essa sia ruotata attorno ad un punto fisso detto "centro di rotazione".

Inoltre, per ciascuna funzione, è stata evidenziata la regione di spazio su cui è avvenuta la ricerca dell'ottimo, le coordinate ottime $\left(x_{1}^{O P T}, x_{2}^{O P T}\right)$ ed il valore ottimo della funzione obiettivo, ovvero $f\left(x^{O P T}\right)$.

\begin{tabular}{|c|c|c|c|c|c|c|c|c|c|}
\hline ID function & Multimodal & Shifted & Separable & Scalable & Rotated & Region & $x_{1}^{O P T}$ & $x_{2}^{O P T}$ & $f\left(x^{O P T}\right)$ \\
\hline 1 & yes & yes & yes & yes & no & {$[-100,100]^{2}$} & $-39,3119$ & 58,8999 & -450 \\
\hline 2 & yes & yes & no & yes & no & {$[-100,100]^{2}$} & 35,6267 & $-82,912$ & -450 \\
\hline 3 & no & yes & no & yes & yes & {$[-100,100]^{2}$} & $-32,2013$ & 64,9776 & -450 \\
\hline 4 & no & yes & no & yes & no & {$[-100,100]^{2}$} & 35,6267 & $-82,912$ & -450 \\
\hline 5 & no & no & no & yes & no & {$[-100,100]^{2}$} & $-5,5559$ & 7,947 & -310 \\
\hline 6 & yes & yes & no & yes & no & {$[-100,100]^{2}$} & 81,0232 & $-48,395$ & 390 \\
\hline 7 & yes & yes & no & yes & yes & {$[0,600]^{2}$} & $-276,268$ & $-11,911$ & -180 \\
\hline 8 & yes & yes & no & yes & no & {$[-32,32]^{2}$} & $-16,823$ & 14,9769 & -140 \\
\hline 9 & yes & yes & yes & yes & no & {$[-5,5]^{2}$} & 1,9005 & $-1,5644$ & -330 \\
\hline 10 & yes & yes & no & yes & yes & {$[-5,5]^{2}$} & 1,9005 & $-1,5644$ & -330 \\
\hline 11 & yes & yes & no & yes & yes & {$[-0.5,0.5]^{2}$} & $-0,1367$ & 0,1186 & 90 \\
\hline 12 & yes & yes & no & yes & no & {$[-\pi,-\pi]^{2}$} & $-2,028$ & $-1,5589$ & -460 \\
\hline 13 & yes & yes & no & yes & no & {$[-5,5]^{2}$} & 0,2471 & $-0,8497$ & -130 \\
\hline 14 & yes & yes & no & yes & no & {$[-100,100]^{2}$} & $-73,6029$ & $-23,55$ & -300 \\
\hline
\end{tabular}

Tabella 4. Caratteristiche delle funzioni benchmark CEC05 impiegate per la validazione delle euristiche globali

Data la natura intrinsecamente stocastica delle euristiche di ricerca dell'ottimo ispirate al mondo naturale, ciascuna tecnica è stata applicata più volte ad ognuna delle funzioni, in modo da poter ragionare in termini di valori medi e di dispersione intorno al valore atteso. In particolare, il numero di replicazioni che si è ritenuto fissare in modo ragionevole su base sperimentale è pari a $M=5$. 
Tutti gli algoritmi sono stati settati imponendo un criterio di stop all'ottimizzazione una volta raggiunto il valore limite di 10.000 function evaluations, ovvero valutazioni della funzione obiettivo. Si è scelto questo numero limitato di fval in modo tale da non assicurarsi solamente la corretta convergenza del metodo, ma soprattutto per capire quale tra le euristiche fosse dotata di una maggiore velocità di tensione verso l'obiettivo. Per ciascuna tecnica è stata creata una tabella con i risultati derivanti dall'applicazione della determinata euristica alle 14 funzioni benchmark considerate. Per quanto riguarda le colonne, la prima mostra l'identificativo numerico (1-14) relativo alle funzioni trattate. La seconda e la terza colonna riportano, per ciascuna funzione, il valore medio rispettivamente per la prima e la seconda componente, calcolato come media aritmetica dei 5 valori ottenuti in corrispondenza delle 5 ottimizzazioni distinte. La quarta colonna indica l'errore medio commesso, inteso come distanza euclidea media (Norma 2) dal reale punto di ottimo, ovvero:

$$
\operatorname{error} x^{A V G}=\frac{1}{M} \sum_{i=1}^{M} \sqrt{\left(x_{1}^{i}-x_{1}^{O P T}\right)^{2}+\left(x_{2}^{i}-x_{2}^{O P T}\right)^{2}}
$$

La quinta colonna presenta lo scarto quadratico medio relativo all'errore commesso nell'individuazione del punto di ottimo. Infine, le ultime tre colonne delle tabelle indicano rispettivamente la media del valore che la funzione assume nel punto che la tecnica ha individuato come "ottimo", l'errore commesso dall'euristica in termini di scostamento medio dal valore reale ottimo della funzione considerata e lo scarto quadratico medio dei 5 valori individuati dalla metodologia in questione.

Le Tabelle 5-11 riportano i risultati ottenuti per ciascuna tecnica 5 .

\begin{tabular}{|c|c|c|c|c|c|c|c|}
\hline ID function & $x_{1}^{A V G}$ & $x_{2}^{A V G}$ & error $^{A V G}$ & $s t . d e v\left(x^{A V G}\right)$ & $f^{A V G}$ & error $f^{A V G}$ & $s t . d e v\left(f^{A V G}\right)$ \\
\hline 1 & $-39,3120$ & 58,8998 & 0,0002 & 0,0001 & $-450,0000$ & 0,0000 & 0,0000 \\
\hline 2 & 32,6259 & $-82,9112$ & 0,0013 & 0,0010 & $-450,0000$ & 0,0000 & 0,0000 \\
\hline 3 & $-18,5926$ & 62,5028 & 8,2628 & 4,9004 & 432,7153 & 539,4966 & 1437,8947 \\
\hline 4 & 35,6319 & $-82,9166$ & 0,0102 & 0,0167 & $-450,0000$ & 0,0000 & 0,0000 \\
\hline 5 & 99,9995 & 99,9997 & 131,8837 & 0,0002 & $-309,7870$ & $-0,2129$ & 0,3788 \\
\hline 6 & 80,4646 & $-22,2969$ & 12,2644 & 15,8126 & 417,2816 & 27,2816 & 45,6024 \\
\hline 7 & $-273,6180$ & $-11,4733$ & 14,2407 & 9,9981 & $-179,8550$ & $-0,1446$ & 0,1776 \\
\hline 8 & $-15,2839$ & 3,8529 & 15,9732 & 2,3055 & $-128,7430$ & $-11,2573$ & 8,6820 \\
\hline 9 & 1,9006 & $-1,5644$ & 0,0002 & 0,0001 & $-330,0000$ & 0,0000 & 0,0000 \\
\hline 10 & 2,2520 & $-1,3391$ & 0,4176 & 0,3811 & $-329,4030$ & $-0,5970$ & 0,5450 \\
\hline 11 & $-0,2490$ & 0,0549 & 0,1580 & 0,2151 & 90,1857 & 0,1857 & 0,1014 \\
\hline 12 & $-2,3260$ & $-0,8309$ & 0,5987 & 0,8193 & $-460,0000$ & $-0,0004$ & 0,0009 \\
\hline 13 & $-0,1382$ & $-1,1632$ & 0,4185 & 0,3788 & $-129,9880$ & $-0,0124$ & 0,0085 \\
\hline 14 & $-73,8819$ & $-23,8148$ & 2,0044 & 0,7603 & $-299,9800$ & $-0,0195$ & 0,0003 \\
\hline
\end{tabular}

Tabella 5. Risultati della Harmony Search (HS)

\begin{tabular}{|c|c|c|c|c|c|c|c|}
\hline ID function & $x_{1}^{A V G}$ & $x_{2}^{A V G}$ & ${\text { error } x^{A V G}}^{A V t . d e v}\left(x^{A V G}\right)$ & $f^{A V G}$ & $\operatorname{error} f^{A V G}$ & st.dev $\left(f^{A V G}\right)$ \\
\hline 1 & $-39,3119$ & 58,8999 & $1,92 \mathrm{E}-06$ & $1,03 \mathrm{E}-06$ & $-450,0000$ & 0,0000 & 0,0000 \\
\hline 2 & 35,6267 & $-82,9123$ & $8,96 \mathrm{E}-06$ & $6,47 \mathrm{E}-06$ & $-450,0000$ & 0,0000 & 0,0000 \\
\hline 3 & $-32,2013$ & 64,9776 & $3,30 \mathrm{E}-06$ & $1,14 \mathrm{E}-06$ & $-450,0000$ & 0,0000 & 0,0000 \\
\hline 4 & 35,6267 & $-82,9123$ & $2,69 \mathrm{E}-05$ & $1,88 \mathrm{E}-05$ & $-450,0000$ & 0,0000 & 0,0000 \\
\hline 5 & 100,0000 & 100,0000 & 131,8842 & 0,0000 & $-310,0000$ & 0,0000 & 0,0000 \\
\hline 6 & 81,0491 & $-48,3086$ & 0,3812 & 0,2845 & 390,4800 & 0,4818 & 0,7070 \\
\hline 7 & $-278,0480$ & $-11,3336$ & 1,8713 & 2,4985 & $-179,9999$ & $-0,0008$ & 0,0004 \\
\hline 8 & $-31,9992$ & 14,9795 & 15,1762 & 0,0017 & $-138,4340$ & $-1,5655$ & 1,2837 \\
\hline 9 & 1,9004 & $-1,5643$ & $7,86 \mathrm{E}-05$ & $5,43 \mathrm{E}-05$ & $-330,0000$ & 0,0000 & 0,0000 \\
\hline 10 & 1,9005 & 1,5641 & 0,0006 & 0,0004 & $-329,9990$ & $-0,0014$ & 0,0020 \\
\hline 11 & $-0,2275$ & 0,2100 & 0,2027 & 0,1643 & 90,0160 & 0,0162 & 0,0114 \\
\hline 12 & $-1,8095$ & $-0,6727$ & 0,6803 & 0,9427 & $-460,0000$ & 0,0000 & 0,0000 \\
\hline 13 & 0,2471 & 0,8496 & 0,0005 & 0,0003 & $-130,0000$ & 0,0000 & 0,0000 \\
\hline 14 & $-74,1684$ & $-23,1759$ & 0,8530 & 1,2404 & $-299,9840$ & $-0,0157$ & 0,0052 \\
\hline
\end{tabular}

Tabella 6. Risultati del Cuckoo Search (CS)

${ }^{5}$ Al fine della validazione degli algoritmi di ottimizzazione scritti dagli autori in linguaggio Matlab sono state riportate tutti i risultati sperimentali, affinchè questi possano essere scientificamente riprodotti o testati su altri case-studies. 


\begin{tabular}{|c|c|c|c|c|c|c|c|}
\hline ID function & $x_{1}^{A V G}$ & $x_{2}^{A V G}$ & $\operatorname{error}^{A V G}$ & $\operatorname{st.dev}\left(x^{A V G}\right)$ & $f^{A V G}$ & $\operatorname{error}^{A V G}$ & $\operatorname{st.dev}\left(f^{A V G}\right)$ \\
\hline 1 & $-39,3120$ & 58,8989 & 0,0010 & $1,25 \mathrm{E}-03$ & $-450,0000$ & 0,0000 & 0,0000 \\
\hline 2 & 35,6265 & $-82,9124$ & 0,0004 & 0,0004 & $-450,0000$ & 0,0000 & 0,0000 \\
\hline 3 & $-6,4729$ & 60,3014 & 26,7578 & 20,1535 & 2787,9267 & 2517,9187 & 3346,3352 \\
\hline 4 & 35,6268 & $-82,9123$ & 0,0003 & 0,0001 & $-450,0000$ & 0,0000 & 0,0000 \\
\hline 5 & 100,0000 & 100,0000 & 131,8842 & 0,0000 & $-310,0000$ & 0,0000 & 0,0000 \\
\hline 6 & 81,0277 & $-48,3857$ & 0,0038 & 0,0040 & 390,0038 & 0,0256 & 0,0188 \\
\hline 7 & $-273,6510$ & $-13,4034$ & 3,4070 & 3,8227 & $-179,9870$ & $-0,0131$ & 0,0152 \\
\hline 8 & $-28,9256$ & 6,5021 & 14,7273 & 0,9169 & $-135,4750$ & $-4,5252$ & 8,7261 \\
\hline 9 & 1,9010 & $-1,5558$ & 0,0086 & 0,0192 & $-330,0000$ & 0,0000 & 0,0000 \\
\hline 10 & 1,7850 & $-1,6383$ & 0,1413 & 0,3101 & $-329,7930$ & $-0,2070$ & 0,4408 \\
\hline 11 & $-0,0671$ & $-0,1279$ & 0,2861 & 0,2182 & 90,0000 & 0,0000 & 0,0000 \\
\hline 12 & $-1,8095$ & $-0,6727$ & 0,6804 & 0,9428 & $-460,0000$ & 0,0000 & 0,0000 \\
\hline 13 & $-0,3049$ & $-1,4212$ & 0,6425 & 0,5481 & $-129,9520$ & $-0,0480$ & 0,0722 \\
\hline 14 & $-76,4639$ & $-20,5758$ & 4,1549 & 4,3809 & $-299,9370$ & $-0,0626$ & 0,1074 \\
\hline
\end{tabular}

Tabella 7. Risultati della Particle Swarm Optimization (PSO)

\begin{tabular}{|c|c|c|c|c|c|c|c|}
\hline ID function & $x_{1}^{A V G}$ & $x_{2}^{A V G}$ & error $^{A V G}$ & st.dev $\left(x^{A V G}\right)$ & $f^{A V G}$ & error $f^{A V G}$ & st.dev $\left(f^{A V G}\right)$ \\
\hline 1 & $-38,3119$ & 58,8999 & $3,62 \mathrm{E}-07$ & 0,0000 & $-450,0000$ & 0,0000 & 0,0000 \\
\hline 2 & 35,6267 & $-82,9123$ & 0,0000 & 0,0000 & $-450,0000$ & 0,0000 & 0,0000 \\
\hline 3 & $-38,0947$ & 66,0442 & 5,9891 & 5,1161 & $-346,5404$ & $-103,4596$ & 0,0006 \\
\hline 4 & 35,5618 & $-82,8741$ & 0,0809 & 0,0478 & $-449,9987$ & $-0,0013$ & 0,0006 \\
\hline 5 & 100,0000 & 100,0000 & 131,8842 & 0,0000 & $-310,0000$ & 0,0000 & 0,0000 \\
\hline 6 & 80,3540 & $-48,9668$ & 1,4026 & 0,1463 & 390,9209 & 0,9209 & 0,6576 \\
\hline 7 & $-275,3580$ & $-11,8758$ & 3,0995 & 2,1429 & $-179,9910$ & $-0,0085$ & 0,0072 \\
\hline 8 & $-31,9667$ & 15,0924 & 15,1451 & 0,0463 & $-137,3730$ & $-2,6270$ & 1,6616 \\
\hline 9 & 1,9005 & 1,5644 & $4,45 \mathrm{E}-07$ & $2,45 \mathrm{E}-07$ & $-330,0000$ & 0,0000 & 0,0000 \\
\hline 10 & 1,9002 & $-1,5650$ & 0,0008 & 0,0007 & $-330,0000$ & 0,0000 & 0,0000 \\
\hline 11 & $-0,0248$ & 0,1822 & 0,3266 & 0,1894 & 90,0796 & 0,0796 & 0,0382 \\
\hline 12 & $-2,2555$ & 0,4189 & 1,5784 & 0,1836 & $-459,9970$ & $-0,0025$ & 0,0009 \\
\hline 13 & 0,2519 & $-0,8455$ & 0,0087 & 0,0150 & $-130,0000$ & 0,0000 & 0,0000 \\
\hline 14 & $-72,4078$ & $-24,3152$ & 1,9071 & 0,5930 & $-299,9990$ & $-0,0242$ & 0,0163 \\
\hline
\end{tabular}

Tabella 8. Risultati dell'Artificial Bee Colony ( $A B C)$

\begin{tabular}{|c|c|c|c|c|c|c|c|}
\hline ID function & $x_{1}^{A V G}$ & $x_{2}^{A V G}$ & error $^{A V G}$ & st.dev $\left(x^{A V G}\right)$ & $f^{A V G}$ & error $f^{A V G}$ & st.dev $\left(f^{A V G}\right)$ \\
\hline 1 & $-38,3119$ & 58,8999 & $4,00 \mathrm{E}-04$ & 0,0002 & $-450,0000$ & 0,0000 & 0,0000 \\
\hline 2 & 35,6267 & $-82,9123$ & $4,00 \mathrm{E}-04$ & 0,0001 & $-450,0000$ & 0,0000 & 0,0000 \\
\hline 3 & $-22,9279$ & 63,2926 & 23,2185 & 9,8929 & 549,3872 & 238,3962 & 307,9016 \\
\hline 4 & 35,6267 & $-82,9122$ & $4,00 \mathrm{E}-04$ & 0,0002 & $-450,0000$ & 0,0000 & 0,0000 \\
\hline 5 & 100,0000 & 100,0000 & 131,8842 & 0,0000 & $-310,0000$ & 0,0000 & 0,0000 \\
\hline 6 & 84,1107 & $-18,3680$ & 9,2359 & 12,8183 & 390,0000 & 0,0000 & 0,0000 \\
\hline 7 & $-274,5150$ & $-12,4780$ & 3,8633 & 3,3802 & $-179,9880$ & $-0,0120$ & 0,0109 \\
\hline 8 & $-5,9739$ & $-4,5164$ & 13,3275 & 5,2611 & $-123,9960$ & $-16,0037$ & 8,9454 \\
\hline 9 & 1,9005 & $-1,5644$ & $1,10 \mathrm{E}-05$ & $6,84 \mathrm{E}-06$ & $-330,0000$ & 0,0000 & 0,0000 \\
\hline 10 & 1,9005 & $-1,5644$ & $1,86 \mathrm{E}-05$ & $7,86 \mathrm{E}-06$ & $-330,0000$ & 0,0000 & 0,0000 \\
\hline 11 & $-0,2032$ & 0,0092 & 0,2385 & 0,1827 & 90,0037 & 0,0037 & 0,0019 \\
\hline 12 & $-1,9724$ & $-0,9687$ & 0,9778 & 0,6114 & $-460,0000$ & 0,0000 & 0,0000 \\
\hline 13 & $-0,1397$ & $-1,3220$ & 0,5151 & 0,7080 & $-129,9920$ & $-0,0080$ & 0,0109 \\
\hline 14 & $-73,2811$ & $-24,0270$ & 1,7621 & 1,6118 & $-299,9999$ & $-0,0098$ & 0,0098 \\
\hline
\end{tabular}

Tabella 9. Risultati del Firefly Algorithm (FA) 


\begin{tabular}{|c|c|c|c|c|c|c|c|}
\hline ID function & $x_{1}^{A V G}$ & $x_{2}^{A V G}$ & error $x^{A V G}$ & st.dev $\left(x^{A V G}\right)$ & $f^{A V G}$ & error $f^{A V G}$ & st.dev $\left(f^{A V G}\right)$ \\
\hline 1 & $-39,3109$ & 58,9040 & 0,0047 & 0,0090 & $-450,0000$ & 0,0000 & 0,0000 \\
\hline 2 & 35,6286 & $-82,915$ & 0,0073 & 0,0052 & $-450,0000$ & 0,0000 & 0,0000 \\
\hline 3 & 7,1738 & 3,5982 & 14,3420 & 6,5350 & 490,5657 & 347,3573 & 606,7839 \\
\hline 4 & 35,6098 & $-82,8672$ & 0,0527 & 0,0536 & $-449,9934$ & $-0,0066$ & 0,0061 \\
\hline 5 & 100,0000 & 100,0000 & 131,8842 & 0,0000 & $-310,0000$ & 0,0000 & 0,0000 \\
\hline 6 & 83,0164 & $-15,8598$ & 19,2852 & 15,6375 & 418,5471 & 28,5470 & 26,8784 \\
\hline 7 & $-283,5640$ & $-13,0591$ & 9,8139 & 12,6055 & $-179,7530$ & $-0,2474$ & 0,2505 \\
\hline 8 & $-12,3215$ & $-9,0158$ & 14,1865 & 3,5301 & $-123,4850$ & $-16,5150$ & 4,7271 \\
\hline 9 & 1,8982 & $-1,5706$ & 0,4047 & 0,5390 & $-329,5580$ & $-0,4420$ & 0,5127 \\
\hline 10 & 1,5419 & $-1,9631$ & 0,5912 & 0,3384 & $-329,2040$ & $-0,7962$ & 0,4445 \\
\hline 11 & 0,0663 & 0,0909 & 0,3282 & 0,0585 & 90,0342 & 0,0342 & 0,0593 \\
\hline 12 & $-1,6745$ & $-1,6967$ & 0,3793 & 0,5194 & $-460,0000$ & 0,0000 & 0,0000 \\
\hline 13 & $-0,3102$ & $-1,4850$ & 0,7200 & 0,5519 & $-129,9760$ & $-0,0240$ & 0,0181 \\
\hline 14 & $-73,0901$ & $-23,4927$ & 6,8571 & 4,2517 & $-299,8230$ & $-0,2042$ & 0,1089 \\
\hline
\end{tabular}

Tabella 10. Risultati della Simulated Annealing (SA)

\begin{tabular}{|c|c|c|c|c|c|c|c|}
\hline ID function & $x_{1}^{A V G}$ & $x_{2}^{A V G}$ & error $^{A V G}$ & st.dev $\left(x^{A V G}\right)$ & $f^{A V G}$ & error $f^{A V G}$ & st.dev $\left(f^{A V G}\right)$ \\
\hline 1 & $-39,3125$ & 58,8785 & 0,0915 & 0,0561 & $-449,9900$ & $-0,0101$ & 0,0167 \\
\hline 2 & 35,3359 & $-82,3581$ & 0,6557 & 0,8968 & $-449,5750$ & $-0,7899$ & 0,9554 \\
\hline 3 & 30,7078 & 53,5464 & 12,1171 & 0,4379 & 3661,5400 & 3211,5400 & 524,6124 \\
\hline 4 & $-0,1122$ & $-4,2558$ & 85,9552 & 4,1651 & 2742,9400 & 2292,9400 & 386,2166 \\
\hline 5 & 99,9992 & 82,3703 & 120,4092 & 5,5013 & 183,5967 & $-84,6113$ & 203,6819 \\
\hline 6 & 70,8787 & 34,2843 & 17,6662 & 4,2691 & 492,9664 & 102,9664 & 6,1568 \\
\hline 7 & 0,5640 & $-0,7556$ & 274,7345 & 0,6946 & $-141,3460$ & $-38,6144$ & 0,8361 \\
\hline 8 & 0,6156 & 0,0850 & 21,7654 & 0,3691 & $-119,9460$ & $-20,0540$ & 0,0350 \\
\hline 9 & 1,3035 & $-0,3704$ & 1,2632 & 0,2486 & $-327,8100$ & $-2,1900$ & 1,6370 \\
\hline 10 & 1,6255 & 0,1179 & 1,1842 & 0,4656 & $-324,6330$ & $-5,3670$ & 3,1927 \\
\hline 11 & 0,2725 & 0,3509 & 0,3117 & 0,1662 & 91,0540 & 1,0540 & 0,6588 \\
\hline 12 & $-0,1442$ & 0,8858 & 2,0033 & 0,2184 & $-460,0000$ & 0,0000 & 0,0000 \\
\hline 13 & 0,4018 & $-0,7985$ & 0,1648 & 0,1668 & $-129,7760$ & $-0,2240$ & 0,4465 \\
\hline 14 & 0,0092 & 0,3092 & 76,9386 & 0,1855 & $-299,0130$ & $-0,9874$ & 0,0211 \\
\hline
\end{tabular}

Tabella 11. Risultati dei Genetic Algorithms (GA)

Osservando i risultati legati alla validazione delle routine di ricerca globale, si nota che le euristiche sono state in grado, nonostante il limitato budget di function evaluations messo a disposizione per tali test comparativi, ad individuare in modo soddisfacente la regione dell'ottimo. Tra le metodologie di ricerca, si sono distinte la Cuckoo Search e l'Artificial Bee Colony ${ }^{6}$.

Laddove lo scarto quadratico medio st.dev $\left(f^{A V G}\right)$ è risultato superiore all'unità, è stato sufficiente aumentare il budget di ulteriori 5.000 lanci per avere la controprova dell'individuazione corretta del punto di minimo.

\section{Risoluzione del problema algoritmico}

In questo paragrafo si procede alla sostituzione del motore di ricerca locale gradientale impiegato nel problema descritto nella sezione 2 del paper con le tecniche globali descritte precedentemente [14].

Al fine di analizzare come tutte le tecniche testate individuino la corretta regione di ottimo della superficie $L(\alpha, \sigma)$, si è effettuata, in accordo con la Response Surface Methodology [15], la grigliatura dei fattori nel dominio sperimentale delimitato dai valori $\alpha \in\left[10^{-6}, 0.03\right]$ e $\sigma \in\left[10^{-6}, 0.25\right]$.

${ }^{6}$ Tale affermazione deriva dall'analisi del confronto numerico tra le tabelle 5-11: si osserva che a fronte di un lieve aumento del tempocomputer un miglioramento medio delle performance. 
Nella Figura 8 è riportato l'intorno più promettente di $L(\alpha, \sigma)$, in corrispondenza del quale si perviene ai migliori valori calibrati per l'albero stocastico di Hull-White.

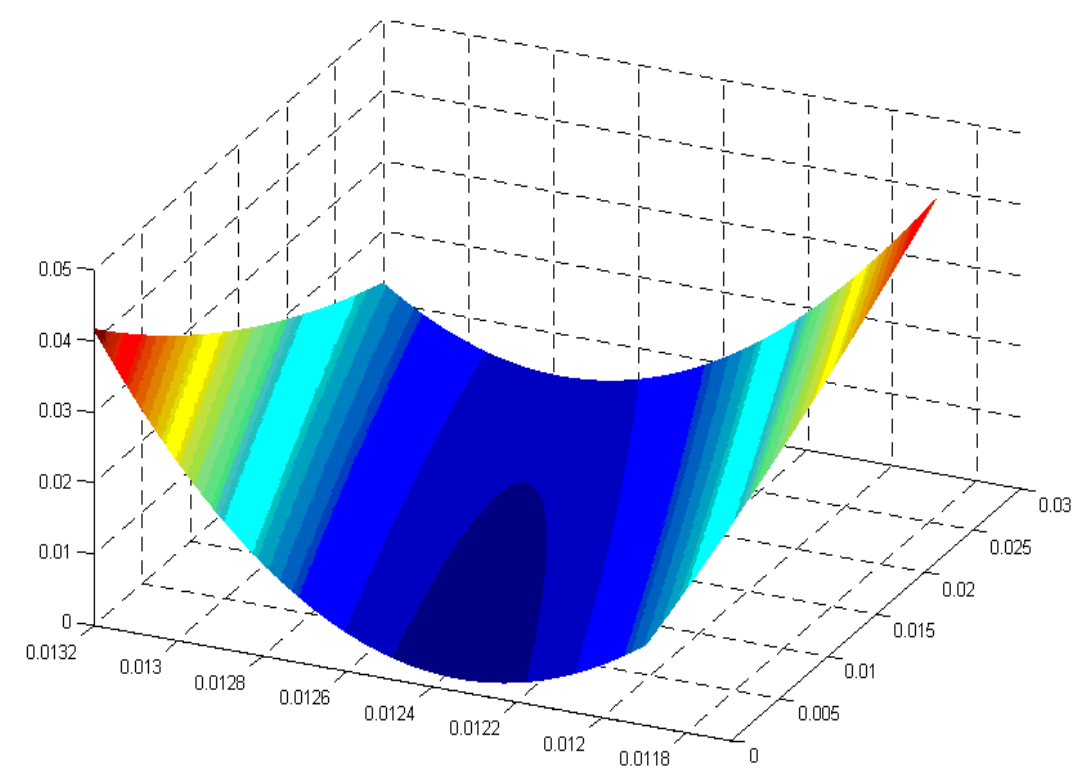

Figura 8. Intorno della zona dell'ottimo per la superficie $L(\alpha, \sigma)$

Come riportato nella Tabella 12, tutte le metodologie di ottimizzazione sono riuscite ad individuare i corretti valori per $\mathrm{i}$ parametri, impiegando un numero di iterazioni inferiore a 10.000.

\begin{tabular}{|c|c|c|c|c|}
\hline Global Heuristic & $\alpha$ & $\sigma$ & $L(\alpha, \sigma)$ & Computational Time [s] \\
\hline ABC & $1,00 \mathrm{E}-06$ & 0,0123658 & 0,0018794 & 20,00 \\
\hline CS & $1,00 \mathrm{E}-06$ & 0,0123405 & 0,0018470 & 4,60 \\
\hline FA & $1,00 \mathrm{E}-06$ & 0,0123406 & 0,0018470 & 25,00 \\
\hline HS & $3,00 \mathrm{E}-06$ & 0,0123549 & 0,0018575 & 10,10 \\
\hline GA & $1,95 \mathrm{E}-04$ & 0,0123351 & 0,0018594 & 2,70 \\
\hline SA & $5,41 \mathrm{E}-04$ & 0,0123629 & 0,0018827 & 4,79 \\
\hline PSO & $1,00 \mathrm{E}-06$ & 0,0123405 & 0,0018470 & 0,40 \\
\hline
\end{tabular}

Tabella 12. Performance delle Euristiche di ricerca globale nella calibrazione dell'albero di Hull-White

\section{Applicazione delle euristiche di ricerca ad un caso recente di mercato}

Questa ultima parte di articolo si propone di applicare il metodo di calibrazione analizzato ad un caso pratico osservato nel mercato americano. I dati d'ingresso del modello sono stati scaricati da Bloomberg ® alla data del 24/08/2015.

La superficie quotata di volatilità dei Cap con tenore trimestrale è riportata nella Figura 9 e la curva free-risk in dollari è riportata nella Tabella 13.

Le caratteristiche finanziarie del cap di riferimento per la calibrazione sono così riassunte:

- Settle Date: $27 / 08 / 2015$

- Maturity Date: $27 / 08 / 2020$

- $A T M$ (At-the-Money) Strike Price: $1.46073 \%$

- Frequency Reset: Trimestrale

- Principal: $1000 \$$

- Day Basis: ACT/360 


\section{$\langle$ HELP> for explanation.}

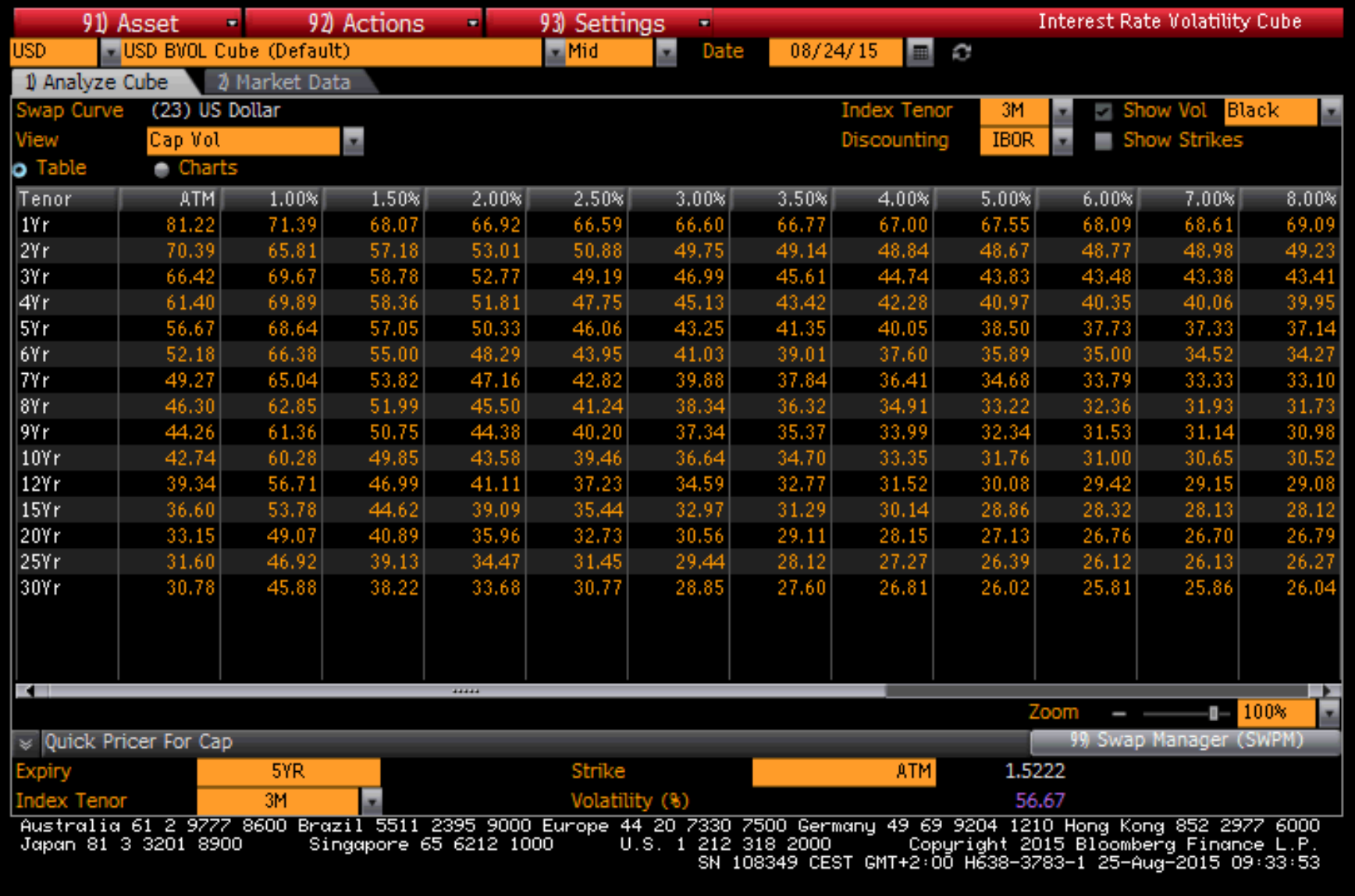

Figura 9. Superficie delle volatilità implicite di Black con tenore trimestrale (Dati di mercato del 24/08/2015)

\begin{tabular}{|c|c|c|c|c|c|}
\hline Term & Curve Horizon & Par-Rate Type & Market Rate & Zero Rate & Discount Factor \\
\hline 3 MO & Short End & Cash Rates & 0,32910 & 0,32910 & 0,999151 \\
\hline EDU5 & Middle & Future SEP15+3 & 0,32487 & 0,33174 & 0,998988 \\
\hline EDV5 & Middle & Future OCT15+3 & 0,35458 & 0,35223 & 0,998593 \\
\hline EDX5 & Middle & Future NOV15+3 & 0,38929 & 0,36901 & 0,998250 \\
\hline EDZ5 & Middle & Future DEC15+3 & 0,41396 & 0,3709 & 0,997943 \\
\hline EDF6 & Middle & Future JAN16+3 & 0,44848 & 0,39126 & 0,997463 \\
\hline EDG6 & Middle & Future FEB16+3 & 0,48803 & 0,41007 & 0,997023 \\
\hline EDH6 & Middle & Future MAR16+3 & 0,53754 & 0,42604 & 0,996589 \\
\hline EDM6 & Middle & Future JUN16+3 & 0,69050 & 0,49568 & 0,994720 \\
\hline EDU6 & Middle & Future SEP16+3 & 0,86802 & 0,56818 & 0,992542 \\
\hline EDZ6 & Middle & Future DEC16+3 & 1,05032 & 0,64078 & 0,990115 \\
\hline EDH7 & Middle & Future MAR17+3 & 1,20176 & 0,72682 & 0,986887 \\
\hline EDM7 & Middle & Future JUN17+3 & 1,35289 & 0,80552 & 0,983523 \\
\hline EDU7 & Middle & Future SEP17+3 & 1,48361 & 0,88067 & 0,979849 \\
\hline EDZ7 & Middle & Future DEC17+3 & 1,60390 & 0,95201 & 0,975892 \\
\hline EDH8 & Middle & Future MAR18+3 & 1,70376 & 1,02157 & 0,971707 \\
\hline EDM8 & Middle & Future JUN18+3 & 1,80321 & 1,08813 & 0,967298 \\
\hline 4 YR & Long End & Swap Rates & 1,29100 & 1,29959 & 0,949470 \\
\hline 5 YR & Long End & Swap Rates & 1,48400 & 1,49790 & 0,928100 \\
\hline 6 YR & Long End & Swap Rates & 1,65000 & 1,67010 & 0,905027 \\
\hline
\end{tabular}

Tabella 13. Struttura a termine dei tassi free-risk in USD (Dati di mercato del 24/08/2015) 
Analogamente al caso precedente, si è effettuata la grigliatura dei fattori per la superficie $L(\alpha, \sigma)$ sul dominio sperimentale $\alpha \in\left[10^{-6}, 0.03\right]$ e $\sigma \in\left[10^{-6}, 0.25\right]$.

L'intorno della zona di ottimo, che permette di avere i migliori valori per $\alpha$ e $\sigma$ è evidenziato in Figura 10 [16].

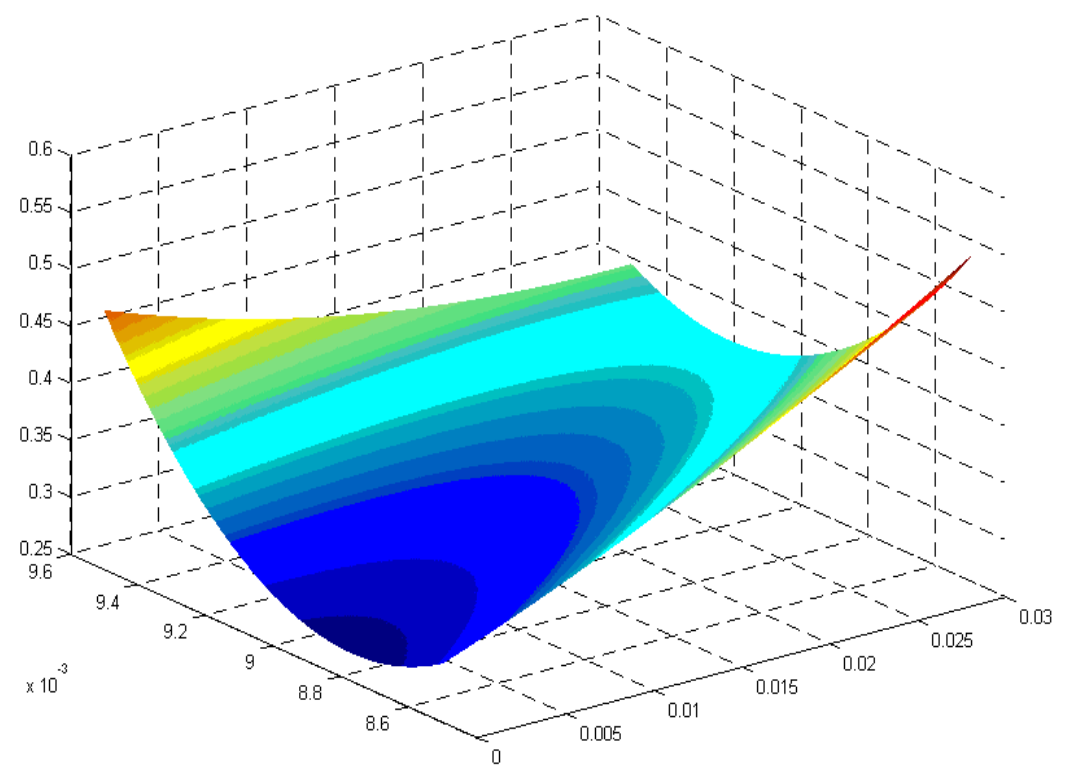

Figura 10. Intorno della zona dell'ottimo per la superficie $L(\alpha, \sigma)$

Come testimonia la Tabella 14, tutte le euristiche sono state in grado di calcolare i valori ottimali dei parametri liberi impiegati dall'albero Hull-White, che permettono di minimizzare la differenza tra il valore teorico e quello osservato dal mercato in un numero massimo di funzioni di valutazione pari a 10.000 .

\begin{tabular}{|c|c|c|c|c|}
\hline Global Heuristic & $\alpha$ & $\sigma$ & $L(\alpha, \sigma)$ & Computational Time [s] \\
\hline ABC & $1,00 \mathrm{E}-06$ & 0,0087896 & 0,2750821 & 20,80 \\
\hline CS & $1,00 \mathrm{E}-06$ & 0,0087702 & 0,2749417 & 4,64 \\
\hline FA & $1,00 \mathrm{E}-06$ & 0,0087703 & 0,2749417 & 12,50 \\
\hline HS & $5,05 \mathrm{E}-05$ & 0,0087710 & 0,2750850 & 10,19 \\
\hline GA & $5,47 \mathrm{E}-05$ & 0,0087912 & 0,2765884 & 1,39 \\
\hline SA & $1,73 \mathrm{E}-04$ & 0,0087808 & 0,2768005 & 2,80 \\
\hline PSO & $1,00 \mathrm{E}-06$ & 0,0087700 & 0,2762778 & 0,32 \\
\hline
\end{tabular}

Tabella 14. Performance delle Euristiche di ricerca globale nella calibrazione dell'albero di Hull-White

\section{Conclusioni}

L'articolo ha evidenziato come nella calibrazione degli alberi stocastici che descrivono l'evoluzione dei tassi d'interesse non sempre l'utilizzo di metodologie di ricerca locale siano idonee ad individuare i valori ottimali dei parametri che caratterizzano la catena di Markov di riferimento. In particolare si è dimostrato come l'implementazione di una euristica di ricerca globale possa aumentare l'affidabilità delle soluzioni che minimizzano la discrepanza tra il modello teorico e il dato di mercato, con un limitato tempo computazionale.

Pier Giuseppe Giribone, Simone Ligato e Simone Fioribello 


\section{Bibliografia}

[1] The MathWorks, "Matlab Financial Instruments User Manual", 2015.

[2] S. Fioribello, P. G: Giribone, S. Ligato, "Proposal and validation of a new heuristic applied to the calibration of stochastic interest-rate trees: the Attraction Force Optimization (AFO) ", AIFIRM Magazine, vol. 9, n.4, 2015, pp.22-34.

[3] J. Hull, A. White, "Numerical Procedures for Implementing Term Structure Models", The Journal of Derivatives, 1994

[4] D. Brigo, F. Mercurio, "Interest Rate Models: Theory and Practice with Smile, Inflation and Credit", Springer, 2006

[5] K. M. Mullen, "Continuous global optimization in R", Journal of Statistical Software, vol. 60, issue 6, 2014.

[6] Z. W. Geem, J. H. Kim, G. V. Loganathan, "A new heuristic Optimization Algorithm: Harmony Search", Simulation, vol. 76, 2001, pp. 60-68.

[7] X. S. Yang, "Cuckoo Search via Levi flights", World Congress on Nature \& Biologically Inspired Computing, IEEE Publications, 2009, pp. 210-214.

[8] J. Kennedy, R. Eberhart, "Particle Swarm Optimization", Proceeding of IEEE International Conference on Neural Networks, pp. 1942-1948, 1995.

[9] D. Dervis Karaboga, "An Idea Based on Honey Bee Swarm for Numerical Optimization", Technical Report TR-06, Erciyes University, Computer Engineering Department, 2005.

[10] X. S. Yang, "Firefly Algorithm, Levy flights and global optimization", Research and Development in Intelligent Systems XXVI, Springer, 2010, pp. 209-218.

[11] J. H. Holland, "Adaptation in Natural and Artificial Systems", MIT Press, 1975.

[12] C. D. Gelatt Jr., M. P. Vecchi, S. Kirkpatrick, "Optimization by Simulated Annealing”, Science, 1983.

[13] A. Auger, Y. P. Chen, K. Deb, N. Hansen, J. J. Liang, P. N. Suganthan, S. Tiwari, "Problems Definition and Evaluation Criteria for the CEC 2005 Special Session on Real-Parameter Optimization", Technical Report, Nanyang Technological University, Singapore, 2005.

[14] Xin-She Yang, "Nature-Inspired Metaheuristic Algorithms - Second Edition", Luniver Press (Univ. of Cambridge), 2010

[15] D. C. Montgomery, "Design of Experiments", John Wiley and Sons, 1997.

[16] Cassettari L., Mosca R., Dispense del corso di Dottorato in Ingegneria Matematica (Università di Genova) - "La Response Surface Methodology negli esperimenti di simulazione" 
Newsletter AIFIRM - Risk Management Magazine

Anno 11, $\mathrm{n}^{\circ} 2$ Aprile - Maggio - Giugno 2016

Direttore Responsabile

Maurizio Vallino

Condirettore

Corrado Meglio

Consiglio Scientifico

Simona Cosma

Paola Ferretti

Giampaolo Gabbi

Andrea Giacomelli

Pier Giuseppe Giribone

Cristiana Schena

Giuseppe Torluccio

Enzo Scannella

Comitato di redazione

Emanuele Diquattro

Fausto Galmarini

Rossano Giuppa

Aldo Letizia

Paolo Palliola

Enzo Rocca

Fabio Salis

Vignettista: Silvano Gaggero

Proprietà, Redazione e Segreteria:

Associazione Italiana Financial Industry Risk Managers (AIFIRM), Via Sile 18, 20139 Milano

Registrazione del Tribunale di Milano n 629 del 10/9/2004

E-mail: segreteria@aifirm.it;

Tel. 3896946315

Lunedì - Venerdì h.15-17

Stampa: Algraphy S.n.c. - Passo Ponte Carrega 62-62r

16141 Genova

Le opinioni espresse negli articoli impegnano unicamente la responsabilità dei rispettivi autori

SPEDIZIONE IN ABBONAMENTO POSTALE AI SOCI AIFIRM RESIDENTI IN ITALIA, IN REGOLA CON L'ISCRIZIONE 


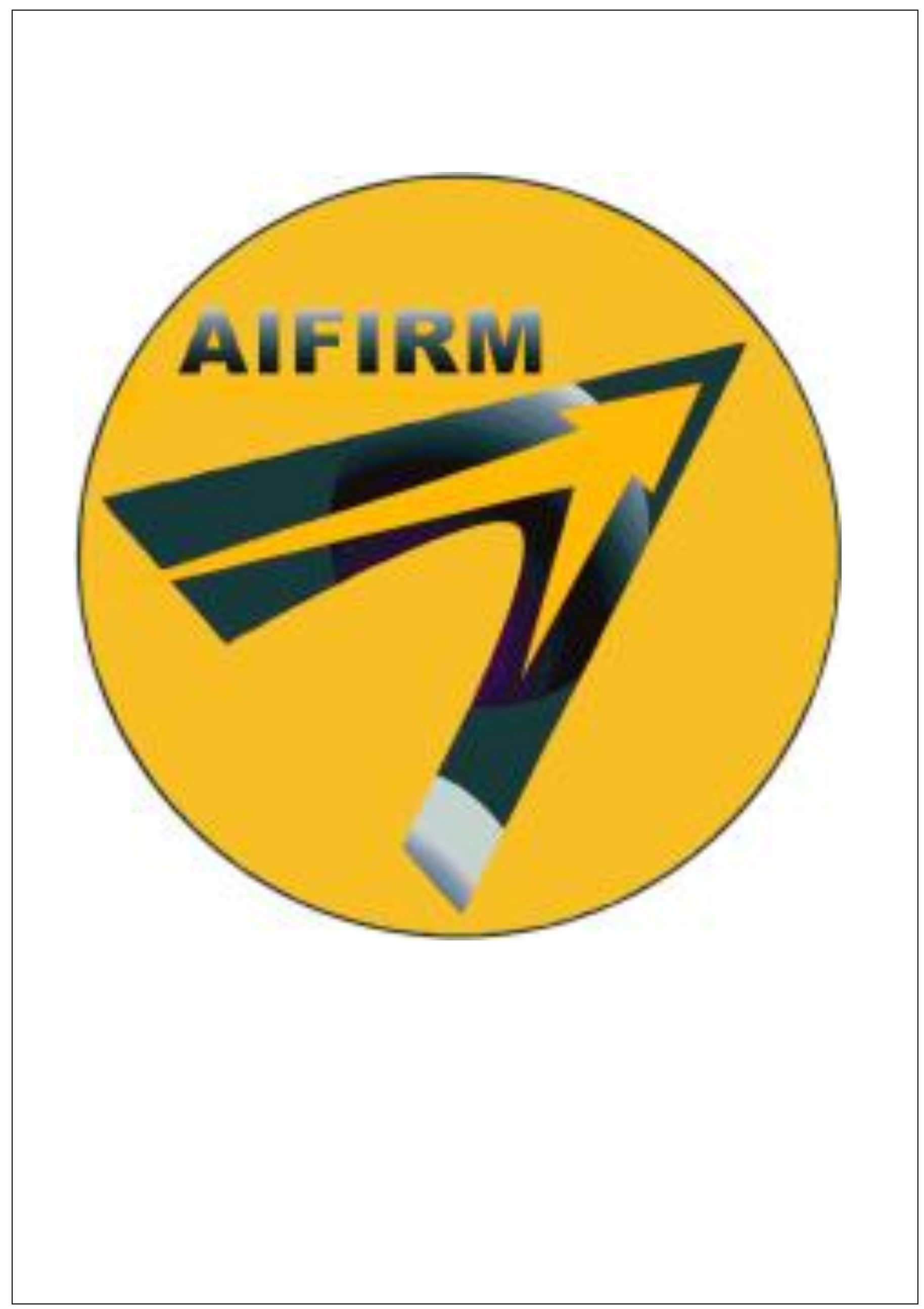

